\title{
Causal optimal transport and its links to enlargement of filtrations and continuous-time stochastic optimization
}

\author{
B. Acciaio* \\ J. Backhoff-Veraguas ${ }^{\dagger}$ \\ A. Zalashko
}

June 4, 2019

\begin{abstract}
The martingale part in the semimartingale decomposition of a Brownian motion with respect to an enlargement of its filtration, is an anticipative mapping of the given Brownian motion. In analogy to optimal transport theory, we define causal transport plans in the context of enlargement of filtrations, as the Kantorovich counterparts of the aforementioned non-adapted mappings. We provide a necessary and sufficient condition for a Brownian motion to remain a semimartingale in an enlarged filtration, in terms of certain minimization problems over sets of causal transport plans. The latter are also used in order to give robust transport-based estimates for the value of having additional information, as well as model sensitivity with respect to the reference measure, for the classical stochastic optimization problems of utility maximization and optimal stopping.
\end{abstract}

Keywords: Causal transport plan; Semimartingale decomposition; Filtration enlargement; Stochastic optimization; Robust bounds; Value of information; Duality.

MSC2010 subject classifications: $91 \mathrm{G} 80,60 \mathrm{G} 44,90 \mathrm{C} 08$.

\section{Introduction}

From the seminal works of Monge [Mon81] and Kantorovich [Kan42], the theory of optimal transport has widely developed and established itself as a fervent research area, with growing applications in the most various areas of sciences and engineering. Powerful connections have also been established between the theory of optimal transport and stochastic analysis, including, among many others, the work by Feyel and Üstünel [FÜ04] extending Brenier's result to Wiener spaces, [BHLP13, GHLT14] on model-independent finance, and [BCH17] on Skorokhod Embedding. In the recent article by Lassalle [Las15], the author creates another bridge between optimal transport and stochastic analysis, considering the transport problem under the so called causality constraint. The origins of this concept can be found in the work of Yamada and Watanabe [YW71]; see also [Jac80, Kur14] for a generalization of the latter. For a discrete-time analogue of transport under causality, see [BBLZ17].

\footnotetext{
*The London School of Economics and Political Science, Dept. Statistics, 10 Houghton St, WC2A 2AE London, UK. Email: b.acciaio@lse.ac.uk

${ }^{\dagger}$ Vienna University of Technology, Institute of Statistics and Mathematical Methods in Economics (E105-7), Wiedner Hauptstraße 8-10, A-1040 Vienna, Austria. Email: julio.backhoff@tuwien.ac.at

${ }^{\ddagger}$ University of Vienna, Faculty of Mathematics, Oskar-Morgenstern-Platz 1, A-1090 Vienna, Austria. Email: anastasiia.zalashko@univie.ac.at. The financial support by the Doktoratskolleg W1245 of the Austrian Science Fund (FWF) and by the Department of Statistics, LSE is gratefully acknowledged.
} 
The aim of the present article is to exploit ideas and techniques from optimal transport under causality, in order to revisit the classical stochastic analysis problem of enlargement of filtrations. We recall that the central question of enlargements of filtrations is whether the semimartingale property is preserved when passing from a given filtration to a larger one; see [BY78, JY78, Jeu80, Jac85] for some of the earliest works on the subject. We also stress that, from the point of view of financial applications, considering different filtrations means accommodating agents having access to different sets of information. This clearly triggers the question of how much having different information matters when facing a particular optimization/decision problem. By means of causal transport, we will address both the issue of semimartingale preservation, as well as that of estimating the value of different (usually additional) information. To describe causality, one is first given two Polish filtered probability spaces $\left(\mathcal{X},\left\{\mathcal{F}_{t}^{\mathcal{X}}\right\}_{t=0}^{T}, \mu\right)$ and $\left(\mathcal{Y},\left\{\mathcal{F}_{t}^{\mathcal{Y}}\right\}_{t=0}^{T}, \nu\right)$. A transport plan $\pi$ is a probability measure on $\mathcal{X} \times \mathcal{Y}$ having the prescribed marginals $\mu, \nu$; this is denoted by $\pi \in \Pi(\mu, \nu)$. It is further called causal if a certain measurability condition holds, roughly: the amount of mass transported by $\pi$ to a subset of the target space $\mathcal{Y}$ belonging to $\mathcal{F}_{t}^{\mathcal{Y}}$, depends on the filtration in the source space only up to time $t$. Thus a causal plan transports $\mu$ into $\nu$ in a non-anticipative way. Although Lassalle analyzes this constrained transport problem in a general set-up, his most noteworthy results (e.g. connection between relative entropy, weak solutions of stochastic differential equations, and causal transports) are obtained in the setting of $\mathcal{X}=\mathcal{Y}$ being the space of continuous functions and, importantly, both filtrations $\mathcal{F}_{t}^{\mathcal{X}}=\mathcal{F}_{t}^{\mathcal{Y}}$ being the canonical one; see [Las15, Sect. 6]. This framework does not allow for anticipation of information, so it is not suitable for the study of enlargement of filtrations.

Given a cost function $c$ on $\mathcal{X} \times \mathcal{Y}$, the general causal transport problem is defined as

$$
\inf \left\{\mathbb{E}^{\pi}[c]: \pi \in \Pi(\mu, \nu), \pi \text { causal }\right\} .
$$

The situation of interest for our purposes is when both $\mathcal{X}$ and $\mathcal{Y}$ are the space of continuous functions, possibly endowed with different filtrations. Concretely, let $B$ be a Brownian motion on some probability space $\left(\Omega, \mathcal{F}^{B}, \mathbb{P}\right)$, where $\mathcal{F}^{B}$ is the filtration generated by $B$, and let $\mathcal{G}^{B} \supseteq \mathcal{F}^{B}$ be a finer filtration (i.e., $\mathcal{G}^{B}$ is an enlargement of $\mathcal{F}^{B}$ ). If $B$ is still a semimartingale with respect to the larger filtration $\mathcal{G}^{B}$, then its unique continuous semimartingale decomposition takes the form

$$
d B_{t}=d \tilde{B}_{t}+d A_{t}
$$

where $\tilde{B}$ is a $\mathcal{G}^{B}$-Brownian motion and $A$ is a continuous $\mathcal{G}^{B}$-adapted finite variation process. Then the joint law of $(\tilde{B}, B)$ turns out to be a causal transport plan on path space, when considering the canonical and an appropriate enlarged filtration (see Section 2.2 for the precise framework).

The main theoretical result of this article is a characterization of the preservation of the semimartingale property in an enlarged filtration, for a process which is a Brownian motion in the original filtration. A necessary and sufficient condition for this preservation property is given in terms of the causal transport problem (1.1) on the space of continuous paths, for specific cost functions depending on the difference of the coordinate processes on the product space; see Theorem 3.2. In addition, when considering transport plans under which this difference is absolutely continuous, we can give necessary and sufficient conditions not only for the semimartingale preservation property to hold, but also to ensure that the finite variation process in (1.2) is absolutely continuous (which yields the so-called information drift); see Theorem 3.5. When the cost function is of Cameron-Martin type, and the filtration enlargement is done entirely at time zero, the causal transport problem can be interpreted in terms of entropy and mutual information. Thus we are inclined to say that, irrespective of the cost function and 
the kind of enlargement, the value of our causal transport problems can be seen as a mutual information in a wider sense.

The analysis contained in this paper is a first step towards the study of the semimartingale preservation problem via optimal transport techniques. In Theorems 3.2 and 3.5, the condition ensuring semimartingale preservation consists in the finiteness of a causal transport problem. As of today, the determination of such a quantity is a barely explored topic. This is one reason why we do not provide in this work new examples where semimartingale preservation holds/fails. On the other hand, at least at an heuristic level, the discretization of this problem could be amenable to existing numerical methods. The growing activity regarding numerical optimal transport, see e.g. [Cut13, $\left.\mathrm{BCC}^{+} 15, \mathrm{PC} 19, \mathrm{EK} 18\right]$, supports this agenda. The study of numerics specific to causal optimal transport in continuous time is object of current research by the authors, while in discrete time there are already results present in the literature, see e.g. [Pfl09, PP12, PP14].

Another contribution of the article consists in the analysis of duality for the primal problem (1.1). Notoriously, duality plays a central role in classical optimal transport. We formulate the causality property via infinitely many linear constraints, which naturally leads to the formulation of a dual problem for (1.1). In order to prove that the values of the primal and dual coincide, we cannot invoke existing results, as these would require imposing restrictive conditions on the problem, and we shall rather take advantage of the specific setting we work in. In the absolutely continuous case described above, we further identify a non-linear dual problem which we can fully solve and relate to the original problem (1.1). This is novel even in the absence of anticipation/enlargements. Interestingly, this gives a different proof of the semimartingale preservation property, and is achieved through optimal transport and convex analysis techniques, without resorting to stochastic analysis arguments; see Theorem 3.8.

We finally describe the main application of the present work. The connection between stochastic analysis and causal transport, as developed in this article, allows us to give a novel application in the framework of continuous-time stochastic optimization. For such problems, we derive what we call robust transport bounds. Concretely, we show how causal transport provides robust estimates, for a class of stochastic optimization problems, regarding both

(i) the value of additional information, and

(ii) model sensitivity.

Point (i) refers to the difference between the values of a stochastic optimization problem when the optimization is run over a smaller "original" filtration, and when it is done over a finer "enlarged" one. We establish that for utility maximization (more generally, stochastic control of linear systems) and optimal stopping, this difference is bounded in a robust way by the value of a causal transport problem; see Proposition 4.8 and Proposition 4.4(i). On the other hand, Point (ii) refers to the difference between the values of a stochastic optimization problem when the optimization is run under two different probabilistic models (i.e. reference probability measures). As in the previous case, we establish that such difference is dominated by a causal transport problem in a rather robust fashion; see Proposition 4.4(ii). We refer to [PK96] for the original motivation regarding the value of information, and to [Pfl09, PP12] for a discrete-time approach related to ours. In specific situations, the computation of our robust bounds can be carried out by PDE methods or discretization schemes; see respectively [BNT19] and [ABC18].

The article is organized as follows. In Section 2 we introduce the main concepts and present some preliminary results. In Section 3 we state and prove our main results on the semimartingale preservation property, we state a non-linear duality result, and provide some links to the literature. Section 4 contains applications of causal transport to continuous-time stochastic optimization problems. Then Section 5 is devoted to an in-depth study of duality when viewing 
(1.1) as a linear program. Finally, in the appendix we collect some technical results, recall some needed tools, and provide some pending proofs.

Notation. For a Polish space $\mathcal{Z}$, we use $\mathcal{P}(\mathcal{Z})$ to denote the set of Borel probability measures on $\mathcal{Z}$, and endow it with the topology of weak convergence. Given a probability space $(\Omega, \mathcal{H}, \mathbb{P})$ and a measurable map $f: \Omega \rightarrow \mathcal{Z}, f_{\#} \mathbb{P} \in \mathcal{P}(\mathcal{Z})$ denotes the push forward of $\mathbb{P}$ by $f$, that is,

$$
f_{\#} \mathbb{P}(C)=\mathbb{P}\left(f^{-1}(C)\right) .
$$

The symbol $\mathbb{E}^{\mathbb{P}}$ denotes integration w.r.t. $\mathbb{P}$. By $\mathcal{B}(\mathcal{Z})$ we denote the Borel $\sigma$-field on $\mathcal{Z}$, and for any $\sigma$-field $\mathcal{J} \subseteq \mathcal{B}(\mathcal{Z})$ we write $B(\mathcal{Z}, \mathcal{J})$ (resp. $B_{b}(\mathcal{Z}, \mathcal{J})$ ) for the set of all real-valued functions on $\mathcal{Z}$ that are measurable (resp. bounded measurable) w.r.t. $\mathcal{J}$. Furthermore, given a measure $\eta \in \mathcal{P}(\mathcal{Z})$, we adopt the notation

$$
{ }^{\eta} \mathcal{J}:=\text { completion of } \mathcal{J} \text { w.r.t. } \eta \text {. }
$$

The unique extension of $\eta$ to ${ }^{\eta} \mathcal{B}(\mathcal{Z})$ is still denoted by $\eta$. With $\mathcal{C}(\mathcal{Z})$ (resp. $\mathcal{C}_{b}(\mathcal{Z})$ ) we mean the set of all continuous (resp. bounded continuous) real-valued functions defined on $\mathcal{Z}$.

\section{Setting and preliminary results}

\subsection{Classical and causal transport.}

Let $(\mathcal{X}, \mathcal{B}(\mathcal{X}), \mu)$ and $(\mathcal{Y}, \mathcal{B}(\mathcal{Y}), \nu)$ be two Polish probability spaces. We denote by $\Pi(\mu, \nu)$ the subset of elements in $\mathcal{P}(\mathcal{X} \times \mathcal{Y})$ having marginals $\mu$ and $\nu$. The classical optimal transport problem consists in minimizing the cost of transporting the (source) measure $\mu$ to the (target) measure $\nu$, with respect to a given cost function $c: \mathcal{X} \times \mathcal{Y} \rightarrow \mathbb{R} \cup\{+\infty\}$. The transportation is represented mathematically by a measure $\pi \in \Pi(\mu, \nu)$, referred to as "transport plan between $\mu$ and $\nu$, so the minimization problem is formulated as

$$
\inf \left\{\mathbb{E}^{\pi}[c]: \pi \in \Pi(\mu, \nu)\right\} .
$$

This kind of problems have a rich theory, particularly concerning optimality conditions and duality. The latter means the equivalence between (2.1) and the following maximization problem

$$
\sup \left\{\mathbb{E}^{\mu}[\phi]+\mathbb{E}^{\nu}[\psi]: \phi \in \mathcal{C}_{b}(\mathcal{X}), \psi \in \mathcal{C}_{b}(\mathcal{Y}), \phi \oplus \psi \leq c\right\}
$$

where here and throughout the article we write

$$
\phi \oplus \psi \leq c \Longleftrightarrow \phi(x)+\psi(y) \leq c(x, y) \forall x, y .
$$

We do not give an exhaustive list of references on the matter, but rather recommend [Vil03, Kel84] for a sample of results in this direction, going from the cost $c$ being lower semicontinuous, to being finite and Borel measurable (and beyond). For our purposes, we will need to slightly extend some of the well-known results in classical transport to our particular setting; see Section 5.1.

We now proceed to introduce the specific class of transport plans we shall consider in this work. To this end, we fix a finite time horizon $[0, T]$, and endow the Polish spaces $\mathcal{X}$ and $\mathcal{Y}$ with right-continuous filtrations $\mathcal{F}^{\mathcal{X}}=\left(\mathcal{F}_{t}^{\mathcal{X}}\right)_{t \in[0, T]}$ and $\mathcal{F}^{\mathcal{Y}}=\left(\mathcal{F}_{t}^{\mathcal{Y}}\right)_{t \in[0, T]}$, with $\mathcal{F}_{T}^{\mathcal{X}}=\mathcal{B}(\mathcal{X})$ and $\mathcal{F}_{T}^{\mathcal{Y}}=\mathcal{B}(\mathcal{Y})$. As a rule, $x, y$ will denote generic elements of $\mathcal{X}, \mathcal{Y}$, respectively. 
Definition 2.1 (Causal transport plan). A transport plan $\pi \in \Pi(\mu, \nu)$ is called causal between $\left(\mathcal{X}, \mathcal{F}^{\mathcal{X}}, \mu\right)$ and $\left(\mathcal{Y}, \mathcal{F}^{\mathcal{Y}}, \nu\right)$ if, for any $t \in[0, T]$ and any set $A \in \mathcal{F}_{t}^{\mathcal{Y}}$, the map

$$
\mathcal{X} \ni x \mapsto \pi^{x}(A)=\mathbb{E}^{\pi}\left[\mathbb{I}_{\mathcal{X} \times A} \mid \mathcal{F}_{T}^{\mathcal{X}} \otimes\{\emptyset, \mathcal{Y}\}\right](x)
$$

is measurable with respect to ${ }^{\mu} \mathcal{F}_{t}^{\mathcal{X}}$, where $\pi^{x}(d y)$ is any regular conditional kernel of $\pi$ w.r.t. the first coordinate. Denote the set of such plans by $\Pi^{\mathcal{F}^{\mathcal{X}}, \mathcal{F}^{\mathcal{Y}}}(\mu, \nu)$.

Intuitively, the causality property means that the amount of mass transported into a set $A \in \mathcal{F}_{t}^{\mathcal{Y}}$ in the target space, depends on the filtration in the source space only up to time $t$. If we think of filtrations in terms of information, this corresponds to a non-anticipative constraint. This concept goes back to the so-called Yamada-Watanabe criterion (see [YW71]), and has been recently popularized by [Las15]. We stress that the last author actually uses a weaker definition, but gives sufficient conditions for its equivalence with the one given above. These are however too restrictive for the purpose of the present work. In the following remark we collect some useful equivalent characterizations of causality. We recall that, two filtrations $\mathcal{H}^{1} \subseteq \mathcal{H}^{2}$ are said to satisfy the $H$-hypothesis if every square integrable $\mathcal{H}^{1}$-martingale is a square integrable $\mathcal{H}^{2}$-martingale.

Remark 2.2. The set $\Pi^{\mathcal{F}^{\mathcal{X}}, \mathcal{F}^{\mathcal{Y}}}(\mu, \nu)$ is never empty, since it contains the product measure. Notice as well that if $\pi$ is causal between $\left(\mathcal{X}, \mathcal{F}^{\mathcal{X}}, \mu\right)$ and $\left(\mathcal{Y}, \mathcal{F}^{\mathcal{Y}}, \nu\right)$, then $\pi$ is also causal between $\left(\mathcal{X}, \mathcal{F}^{\mathcal{X}}, \mu\right)$ and $\left(\mathcal{Y}, \tilde{\mathcal{F}}^{\mathcal{Y}}, \nu\right)$ for all $\tilde{\mathcal{F}}^{\mathcal{Y}} \subseteq \mathcal{F}^{\mathcal{Y}}$, as seen directly from Definition 2.1.

Remark 2.3. For a probability measure $\pi \in \Pi(\mu, \nu)$, the following are equivalent:

1. $\pi$ is a causal transport plan between $\mathcal{F}^{\mathcal{X}}$ and $\mathcal{F}^{\mathcal{Y}}$;

2. $\pi\left(\mathcal{X} \times D_{t} \mid \mathcal{F}_{t}^{\mathcal{X}} \otimes\{\emptyset, \mathcal{Y}\}\right)=\pi\left(\mathcal{X} \times D_{t} \mid \mathcal{F}_{T}^{\mathcal{X}} \otimes\{\emptyset, \mathcal{Y}\}\right) \pi$-a.s., for all $t \in[0, T], D_{t} \in \mathcal{F}_{t}^{\mathcal{Y}}$;

3. the $\sigma$-fields $\{\emptyset, \mathcal{X}\} \otimes \mathcal{F}_{t}^{\mathcal{Y}}$ and $\mathcal{F}_{T}^{\mathcal{X}} \otimes\{\emptyset, \mathcal{Y}\}$ are conditionally independent with respect to $\pi$ given $\mathcal{F}_{t}^{\mathcal{X}} \otimes\{\emptyset, \mathcal{Y}\}$, for all $t \in[0, T]$;

4. the $H$-hypothesis holds between ${ }^{\mu} \mathcal{F}^{\mathcal{X}} \otimes\{\emptyset, \mathcal{Y}\}$ and ${ }^{\mu} \mathcal{F}^{\mathcal{X}} \otimes \mathcal{F}^{\mathcal{Y}}$ with respect to $\pi$.

The equivalences above can be shown as in [BY78, Theorem 3]. For convenience of the reader we just stress the reason why causality/conditional independence implies the $H$-hypothesis: if $M$ is a $\left(\pi,{ }^{\mu} \mathcal{F}^{\mathcal{X}} \otimes\{\emptyset, \mathcal{Y}\}\right)$-martingale, then $\mathbb{E}^{\pi}\left[M_{t+s} \mid{ }^{\mu} \mathcal{F}_{t}^{\mathcal{X}} \otimes \mathcal{F}_{t}^{\mathcal{Y}}\right]=\mathbb{E}^{\pi}\left[\left.M_{t+s}\right|^{\mu} \mathcal{F}_{t}^{\mathcal{X}} \otimes\{\emptyset, \mathcal{Y}\}\right]=M_{t}$, hence $M$ is a $\left(\pi,{ }^{\mu} \mathcal{F}^{\mathcal{X}} \otimes \mathcal{F}^{\mathcal{Y}}\right)$-martingale.

In analogy with (2.1), and as in [Las15], we define the causal transport problem:

$$
\inf \left\{\mathbb{E}^{\pi}[c]: \pi \in \Pi^{\mathcal{F}^{\mathcal{X}}, \mathcal{F}^{\mathcal{Y}}}(\mu, \nu)\right\},
$$

which constitutes the core of our work. In Section 5 we will prove a duality result for this problem, and in Sections 3 and 4 we will show how the causal transport problem for specific cost functions allows to characterize semimartingale preservation under filtration enlargement, and to estimate the value of additional information for some stochastic optimization problems.

\subsection{Path space and filtration enlargement.}

We will consider causal plans that transport measures defined on spaces of continuous functions. For $t \in(0, T]$, we denote by $\mathcal{C}[0, t]$ the set of continuous functions $f:[0, t] \rightarrow \mathbb{R}$ such that 
$f(0)=0$, and we let $\mathcal{C}=\mathcal{C}[0, T]$. Let $W=\left(W_{t}\right)_{t \in[0, T]}$ be the coordinate process on $\mathcal{C}, W_{t}(\omega)=\omega_{t}$ for $\omega \in \mathcal{C}$, and let $\mathcal{F}=\left(\mathcal{F}_{t}\right)_{t \in[0, T]}$ the right-continuous version of the filtration generated by $W$ :

$$
\mathcal{F}_{t}:=\bigcap_{u>t} \sigma\left(W_{s}, s \leq u\right)
$$

In order to consider all possible kinds of anticipation of information regarding the evolution of the coordinate process, we study right-continuous filtrations $\mathcal{G}=\left(\mathcal{G}_{t}\right)_{t \in[0, T]}$ such that

$$
\mathcal{G}_{t} \supseteq \mathcal{F}_{t} \text { for all } t \in[0, T) \text {, and } \mathcal{G}_{T}=\mathcal{F}_{T} \text {. }
$$

It is worth mentioning the two most studied kinds of filtration enlargements, which are particular cases of $(2.3)$ :

- initial enlargement with a $\left(\mathcal{F}_{T}\right.$-measurable) random variable, say $L$ (so $\mathcal{G}_{t}=\mathcal{F}_{t} \vee \sigma(L)$, $t \in[0, T])$

- progressive enlargement with a random time (non-negative $\mathcal{F}_{T}$-measurable random variable), say $\tau$ (so $\left(\mathcal{G}_{t}\right)$ is the right-continuous version of $\left(\mathcal{G}_{t}^{0}\right)$, where $\mathcal{G}_{t}^{0}:=\mathcal{F}_{t} \vee \sigma(\tau \wedge t$ ), $t \in[0, T])$, in which case $\mathcal{G}$ turns $\tau$ into a stopping time.

In fact, not many works are devoted to the study of general enlargements of filtration beyond these two cases, as considered in the present article, see e.g. [ADI07, Jeu80, KP15, CJ18]. We refer the reader to the monographs [Jeu80, MY06, AJ17], [JYC09, Sec. 5.9] and [Pro04, Ch. VI], for an account of the main results and the literature on filtration enlargements.

In what follows we will consider $\mathcal{X}=\mathcal{Y}=\mathcal{C}$ and, given two measures $\mu, \nu$ on $\mathcal{C}$, we will study causal transport plans between $(\mathcal{C}, \mathcal{F}, \mu)$ and $(\mathcal{C}, \mathcal{G}, \nu)$. We shall commonly denote by

$$
(\omega, \bar{\omega})
$$

generic elements in $\mathcal{C} \times \mathcal{C}$. Often, as source measure $\mu$, we will take

$$
\gamma:=\text { Wiener measure on } \mathcal{C} \text { started at } 0 .
$$

For a continuous process $Z=\left(Z_{t}\right)_{t \in[0, T]}$ defined on a given space $\Omega$, we denote by

$$
\mathcal{F}^{Z}:=Z^{-1}(\mathcal{F}) \text { and } \mathcal{G}^{Z}:=Z^{-1}(\mathcal{G}),
$$

respectively, the right-continuous version of the filtration generated by $Z$ on $\Omega$, and the filtration containing anticipation of information regarding the evolution of $Z$. Working with the path space $\mathcal{C}$ eases the exposition of our analysis. We point out, however, that our results have a natural extension in the multidimensional setting $\mathcal{C}^{d}$, i.e. for multidimensional continuous processes, see Remarks 3.3 and 3.10 .

For the rest of this section, we work on a fixed probability space $(\Omega, \mathcal{H}, \mathbb{P})$. In particular, for any process $Z=\left(Z_{t}\right)_{t \in[0, T]}$ defined on it, it is implicitly understood that $\mathcal{F}_{T}^{Z} \subseteq \mathcal{H}$.

Definition 2.4 (Causal coupling). A pair $(X, Y)$ of continuous processes on $(\Omega, \mathcal{H}, \mathbb{P})$ is called a causal coupling w.r.t. the filtrations $\mathcal{F}^{X}$ and $\mathcal{G}^{Y}$ if $(X, Y)_{\#} \mathbb{P}$ is a causal transport plan between $\left(\mathcal{C}, \mathcal{F}, X_{\#} \mathbb{P}\right)$ and $\left(\mathcal{C}, \mathcal{G}, Y_{\#} \mathbb{P}\right)$.

Remark 2.5. Definition 2.1 and Remark 2.3 imply that $(X, Y)$ is a causal coupling if and only if for all $t, \mathcal{G}_{t}^{Y}$ and $\mathcal{F}_{T}^{X}$ are conditionally independent given $\mathcal{F}_{t}^{X}$, that is, the $H$-hypothesis holds between $\mathcal{F}^{X}$ and $\mathcal{F}^{X} \vee \mathcal{G}^{Y}$, with respect to $\mathbb{P}$. Note also that the concept of causality here corresponds to that of strong global noncausality by Florens and Fougere [FF96]. More precisely, $(X, Y)$ is a causal coupling w.r.t. $\mathcal{F}^{X}$ and $\mathcal{G}^{Y}$ in our sense, if and only if $\left(\mathcal{G}_{t}^{Y}\right)$ does not strongly cause $X$ given $\left(\mathcal{F}_{t}^{X}\right)$ in the sense of [FF96]. 
The following result shows that there is an easier way to check causality in a Brownian setting; it extends the result [Las15, Proposition 4] to our setting with enlargements.

Lemma 2.6. Let $X$ be a Brownian motion, and $Y$ be a continuous process. Then the following are equivalent:

1. $(X, Y)$ is a causal coupling w.r.t. $\mathcal{F}^{X}$ and $\mathcal{G}^{Y}$;

2. $X$ is a Brownian motion w.r.t. $\mathcal{F}^{X} \vee \mathcal{G}^{Y}$;

3. there is a filtration $\mathcal{J}$ on $\Omega$ s.t. $X$ is a $\mathcal{J}$-Brownian motion and $\mathcal{G}^{Y} \subseteq \mathcal{J}$.

From the proof of the lemma, it will become clear that more generally if $X$ is any process such that $X_{t+s}-X_{t}$ is independent of $\mathcal{G}_{t}^{Y} \vee \mathcal{F}_{t}^{X}$ for all $s, t \geq 0$, then $(X, Y)$ is causal.

Proof. $1 \Rightarrow 2$ : By Lévy theorem, it is enough to show that $X$ is an $\mathcal{F}^{X} \vee \mathcal{G}^{Y}$-martingale. For $0 \leq s<t \leq T$ and $f_{s} \in L^{\infty}\left(\Omega, \mathcal{F}_{s}^{X} \vee \mathcal{G}_{s}^{Y}, \mathbb{P}\right)$, we have

$$
\begin{aligned}
\mathbb{E}^{\mathbb{P}}\left[X_{t} f_{s}\right] & =\mathbb{E}^{\mathbb{P}}\left[\mathbb{E}^{\mathbb{P}}\left[X_{t} f_{s} \mid \mathcal{F}_{t}^{X}\right]\right]=\mathbb{E}^{\mathbb{P}}\left[X_{t} \mathbb{E}^{\mathbb{P}}\left[f_{s} \mid \mathcal{F}_{t}^{X}\right]\right]=\mathbb{E}^{\mathbb{P}}\left[X_{t} \mathbb{E}^{\mathbb{P}}\left[f_{s} \mid \mathcal{F}_{s}^{X}\right]\right] \\
& =\mathbb{E}^{\mathbb{P}}\left[\mathbb{E}^{\mathbb{P}}\left[X_{t} \mid \mathcal{F}_{s}^{X}\right] \mathbb{E}^{\mathbb{P}}\left[f_{s} \mid \mathcal{F}_{s}^{X}\right]\right]=\mathbb{E}^{\mathbb{P}}\left[X_{s} \mathbb{E}^{\mathbb{P}}\left[f_{s} \mid \mathcal{F}_{s}^{X}\right]\right]=\mathbb{E}^{\mathbb{P}}\left[X_{s} f_{s}\right],
\end{aligned}
$$

where causality is used in the third equality.

$2 \Rightarrow 3$ follows by taking $\mathcal{J}:=\mathcal{F}^{X} \vee \mathcal{G}^{Y}$.

$3 \Rightarrow 1$ : For $t \in[0, T]$ and $D_{t} \in \mathcal{G}_{t}^{Y}$,

$$
\mathbb{P}\left(D_{t} \mid \mathcal{F}_{T}^{X}\right)=\mathbb{P}\left(D_{t} \mid \mathcal{F}_{t}^{X},\left\{X_{t+u}-X_{t}, u \in[0, T-t]\right\}\right)=\mathbb{P}\left(D_{t} \mid \mathcal{F}_{t}^{X}\right),
$$

since $X$ is an $\mathcal{F}^{X} \vee \mathcal{G}^{Y}$-Brownian motion, hence its increments $X_{t+s}-X_{t}$ are jointly independent of $\mathcal{F}_{t}^{X}$ and of the event $D_{t} \in \mathcal{G}_{t}^{Y}$. This shows that $(X, Y)$ is causal, by Definition 2.4 and Remark 2.3(2).

Throughout the article, we talk of (continuous) semimartingale decomposition, referring to the unique decomposition of a continuous semimartingale into a continuous local martingale and a continuous finite variation process. The notion of causality can be used to study semimartingale decompositions in the setting of enlargement of filtrations, and this is the object of study of Section 3. We start by illustrating in Section 2.2.1 the lemma above, and show a first connection between decomposition of semimartingales and causality. A necessary and sufficient condition for a Brownian motion to remain a semimartingale in the enlarged filtration is given in Theorem 3.2.

\subsubsection{Some examples of enlargement of Brownian filtration}

In this section we collect some well-known examples of filtration enlargements in a Brownian setting, which will be useful for future reference (see e.g. [MY06] for these and many other examples). Let $B$ be a Brownian motion in its right-continuous natural filtration. If $B$ remains a semimartingale with respect to the enlarged filtration $\mathcal{G}^{B}$, then its unique continuous semimartingale decomposition takes the form

$$
d B_{t}=d \tilde{B}_{t}+d A_{t}
$$

where $\tilde{B}$ is a $\mathcal{G}^{B}$-Brownian motion and $A$ is a continuous $\mathcal{G}^{B}$-adapted finite variation process. In particular, for every finite horizon $T>0$, by Lemma 2.6, we have that $(\tilde{B}, B)$ is a causal transport plan w.r.t. $\mathcal{F}^{\tilde{B}}$ and $\mathcal{G}^{B}$, that is, $(\tilde{B}, B)_{\#} \mathbb{P} \in \Pi^{\mathcal{F}, \mathcal{G}}(\gamma, \gamma)$. On the other hand, note that in 
general $(B, \tilde{B})$ is not causal, not even w.r.t. $\mathcal{F}^{B}$ and $\mathcal{F}^{\tilde{B}}$, that is, $(B, \tilde{B})_{\#} \mathbb{P} \notin \Pi^{\mathcal{F}, \mathcal{F}}(\gamma, \gamma)$. For example, this can be easily seen in the bridge case (2) illustrated below, where $d \tilde{B}_{t}=d B_{t}-\frac{B_{T}-B_{t}}{T-t} d t$.

(1) Initial enlargement with countably many atoms (see [Yor85]): initial enlargement with a discrete $\mathcal{F}_{T}^{B}$-measurable random variable, say $L$ (namely $\mathcal{G}_{t}^{B}=\mathcal{F}_{t}^{B} \vee \sigma(L)$ for all $t \in[0, T]$ ), that takes values $l_{n}, n \in \mathbb{N}$. This corresponds to enlarging the filtration at time zero with the sets $C_{n}=\left\{L=l_{n}\right\}, n \in \mathbb{N}$. In this case the decomposition of $B$ in the enlarged filtration takes the form

$$
d B_{t}=d \tilde{B}_{t}+\sum_{n} \mathbb{I}_{C_{n}} \frac{\eta_{t}^{n}}{M_{t}^{n}} d t
$$

where $M_{t}^{n}=\mathbb{P}\left(C_{n} \mid \mathcal{F}_{t}^{B}\right)$, which by martingale representation can be written as $M_{t}^{n}=M_{0}^{n}+$ $\int_{0}^{t} \eta_{s}^{n} d B_{s}$, with $M_{0}^{n}=\mathbb{P}\left(C_{n}\right)$ and for some predictable process $\eta^{n}$.

(2) Brownian bridge: initial enlargement by the value of the Brownian motion at the terminal time $T$, namely $\mathcal{G}_{t}^{B}=\mathcal{F}_{t}^{B} \vee \sigma\left(B_{T}\right)$ for all $t \in[0, T]$. In this case it is well-known that the decomposition of $B$ in the enlarged filtration $\mathcal{G}^{B}=\mathcal{F}^{B} \vee \sigma\left(B_{T}\right)$ is

$$
d B_{t}=d \tilde{B}_{t}+\frac{B_{T}-B_{t}}{T-t} d t .
$$

(3) Progressive enlargement with last hitting time (see [Yor97, Section 12.2.4]): progressive enlargement with the random time $\tau=\sup \left\{u \leq T, B_{u}=0\right\}$. In this case, using the notation $\Phi(x)=\sqrt{2 / \pi} \int_{x}^{\infty} e^{-u^{2} / 2} d u$, the decomposition of $B$ in the enlarged filtration is the following:

$$
d B_{t}=d \tilde{B}_{t}-\sqrt{\frac{2}{\pi}} \frac{\exp \left(-B_{t}^{2} / 2(T-t)\right)}{\sqrt{T-t}}\left(\frac{\operatorname{sgn}\left(B_{t}\right)}{\Phi\left(\left|B_{t}\right| / \sqrt{T-t}\right)} \mathbb{I}_{\{t \leq \tau\}}-\frac{\operatorname{sgn}\left(B_{T}\right)}{1-\Phi\left(\left|B_{t}\right| / \sqrt{T-t}\right)} \mathbb{I}_{\{\tau \leq t \leq T\}}\right) d t .
$$

(4) Bessel process (see [Jeu80, Sect. 6.3]): define a 3-dimensional Bessel process by $d R_{t}=$ $\frac{1}{R_{t}} d t+d B_{t}$, and denote $J_{t}:=\inf _{s \geq t} R_{s}$. Then the process $\tilde{B}_{t}:=R_{t}-2 J_{t}$ is a Brownian motion in the filtration obtained by enlarging $\mathcal{F}^{B}$ with the process $J$, and

$$
d B_{t}=d \tilde{B}_{t}+\left(2 d J_{t}-\frac{1}{R_{t}} d t\right) .
$$

Note that, contrary to the previous cases, here the finite variation process in the semimartingale decomposition of $B$ in the enlarged filtration is not absolutely continuous.

\section{Causal optimal transport and semimartingale decomposition}

Throughout this whole section we consider the continuous path space framework of Section 2.2. In particular, we consider the filtrations $\mathcal{F}$ and $\mathcal{G}$ defined there. The following notation will be frequently used: for a process/path $Z$ we denote by

$$
V_{t}(Z)=\sup _{0 \leq t_{1} \leq \cdots \leq t_{k} \leq t} \sum_{i<k}\left|Z_{t_{i}}-Z_{t_{i+1}}\right|
$$

the variation of $Z$ up to time $t$.

We show the connection between the semimartingale decompositions arising in enlargement of filtrations, and certain causal optimal transport problems. We consider both the case when the finite variation part in the semimartingale decomposition is absolutely continuous and when it is not; we do it separately for the sake of applications and connection with the literature. All results are shown in a one-dimensional Brownian setting, for simplicity of exposition. We note, however, that analogous results hold in a multidimensional setting, see Remarks 3.3 and 3.10. Recall that $\gamma$ is the Wiener measure on the path space $\mathcal{C}$, and that $(\omega, \bar{\omega})$ denotes a generic element in $\mathcal{C} \times \mathcal{C}$. By $i d$ we mean the identity mapping on $\mathcal{C}$. 


\subsection{The general case}

We first need to obtain the following result, reminiscent of [Las15, Proposition 6]:

Theorem 3.1. Let $\nu$ be a measure on $\mathcal{C}$ such that $\nu \ll \gamma$. Then the following are equivalent:

(i) for some continuous, ${ }^{\nu} \mathcal{G}$-adapted, integrable variation process $A=A(\bar{\omega})$, i.e. such that

$$
\mathbb{E}^{\nu}\left[V_{T}(A)\right]<\infty,
$$

the process $\xi_{t}(\bar{\omega}):=\bar{\omega}_{t}-A_{t}(\bar{\omega})$ is a $\left(\nu,{ }^{\nu} \mathcal{G}\right)$-Brownian motion;

(ii) the following causal optimal transport problem is finite:

$$
\inf _{\pi \in \Pi^{\mathcal{F}, \mathcal{G}}(\gamma, \nu)} \mathbb{E}^{\pi}\left[V_{T}(\bar{\omega}-\omega)\right] .
$$

Moreover, whenever (i)-(ii) hold, we have that:

1. the transport plan $\hat{\pi}:=(\xi, i d)_{\#} \nu$ belongs to $\Pi^{\mathcal{F}, \mathcal{G}}(\gamma, \nu)$, and is optimal for (3.2);

2. for every transport plan $\pi \in \Pi^{\mathcal{F}, \mathcal{G}}(\gamma, \nu)$ with finite cost in (3.2), the process $\tilde{A}(\omega, \bar{\omega}):=A(\bar{\omega})$ in (i) is the $(\pi, \pi(\{\emptyset, \mathcal{C}\} \otimes \mathcal{G}))$-dual predictable projection of the process $(\omega, \bar{\omega}) \mapsto \Lambda(\omega, \bar{\omega}):=\bar{\omega}-\omega$.

Proof. $(i) \Rightarrow(i i)$ : From the process $\xi$ in $(i)$, define the coupling $\hat{\pi}:=(\xi, i d)_{\#} \nu$. The fact that $\hat{\pi} \in \Pi^{\mathcal{F}, \mathcal{G}}(\gamma, \nu)$ follows as in the proof of Lemma 2.6, and $\mathbb{E}^{\hat{\pi}}\left[V_{T}(\bar{\omega}-\omega)\right]=\mathbb{E}^{\nu}\left[V_{T}(A)(\bar{\omega})\right]<\infty$.

$(i i) \Rightarrow(i)$ : Fix $\pi \in \Pi^{\mathcal{F}, \mathcal{G}}(\gamma, \nu)$ with $\mathbb{E}^{\pi}\left[V_{T}(\bar{\omega}-\omega)\right]<\infty$. The continuous process $\Lambda_{t}(\omega, \bar{\omega}):=$ $\bar{\omega}_{t}-\omega_{t}$ is of integrable variation with respect to $\pi$, hence we can define its dual predictable projection $\tilde{A}(\omega, \bar{\omega})$ with respect to ${ }^{\pi}(\{\emptyset, \mathcal{C}\} \otimes \mathcal{G})$, the $\pi$-completion of $\{\emptyset, \mathcal{C}\} \otimes \mathcal{G}$. In particular, $\tilde{A}$ is an integrable variation process on $\mathcal{C} \times \mathcal{C}$. By Lemma C.1, we may assume that $\tilde{A}$ does not depend on the first coordinate, thus it corresponds to a ${ }^{\nu} \mathcal{G}$-predictable integrable variation process $A$ on $\mathcal{C}$, in the sense that $\tilde{A}(\omega, \bar{\omega})=A(\bar{\omega})$, which gives (3.1). Altogether we have

$$
\mathbb{E}^{\pi}\left[\int_{0}^{T} X_{t}(\bar{\omega}) d \Lambda_{t}(\omega, \bar{\omega})\right]=\mathbb{E}^{\nu}\left[\int_{0}^{T} X_{t}(\bar{\omega}) d A_{t}(\bar{\omega})\right],
$$

for every ${ }^{\nu} \mathcal{G}$-predictable bounded process $X$. Moreover, we have

$$
\mathbb{E}^{\nu}\left[V_{T}(A)\right]=\mathbb{E}^{\pi}\left[V_{T}(\tilde{A})\right] \leq \mathbb{E}^{\pi}\left[V_{T}(\Lambda)\right]<\infty .
$$

Now, note that the jump times of $A$ are ${ }^{\nu} \mathcal{G}$-predictable, by [DM80, Theorem B, page xiii], and that for each jump time $\tau, \Delta A_{\tau}=\mathbb{E}^{\pi}\left[\Delta \Lambda_{\tau} \mid\{\emptyset, \mathcal{C}\} \otimes^{\nu} \mathcal{G}_{\tau-}\right]=0$ a.s., from the continuity of $\Lambda$; see [DM80, Theorem VI.76]. Therefore, $A$ is continuous.

We now define the process $\xi$ as in $(i)$, and need to show that it is a $\left(\nu,{ }^{\nu} \mathcal{G}\right)$-Brownian motion. For $0 \leq s<t \leq T$ and $f_{s} \in L^{\infty}\left(\mathcal{C},{ }^{\nu} \mathcal{G}_{s}, \nu\right)$, we have

$$
\begin{aligned}
\mathbb{E}^{\nu}\left[\left(\xi_{t}(\bar{\omega})-\xi_{s}(\bar{\omega})\right) f_{s}(\bar{\omega})\right] & =\mathbb{E}^{\nu}\left[\left(\bar{\omega}_{t}-\bar{\omega}_{s}-\int_{s}^{t} d A_{u}(\bar{\omega})\right) f_{s}(\bar{\omega})\right] \\
& =\mathbb{E}^{\pi}\left[\left(\omega_{t}-\omega_{s}\right) f_{s}(\bar{\omega})\right]+\mathbb{E}^{\pi}\left[\left(\int_{s}^{t} d \Lambda_{u}-\int_{s}^{t} d A_{u}(\bar{\omega})\right) f_{s}(\bar{\omega})\right] \\
& =\mathbb{E}^{\pi}\left[\left(\int_{s}^{t} d \Lambda_{u}-\int_{s}^{t} d A_{u}(\bar{\omega})\right) f_{s}(\bar{\omega})\right]=0,
\end{aligned}
$$

where the third equality follows since $\omega$, which is a $\left(\gamma,{ }^{\gamma} \mathcal{F}\right)$-martingale, is consequently by causality a $\left(\pi,{ }^{\gamma} \mathcal{F} \otimes \mathcal{G}\right)$-martingale, thus also a $\left(\pi,{ }^{\pi}\left({ }^{\gamma} \mathcal{F} \otimes \mathcal{G}\right)\right)$-martingale and in particular a $\left(\pi,{ }^{\gamma} \mathcal{F} \otimes^{\nu} \mathcal{G}\right)$ martingale. The last equality follows from (3.3) with $X_{t}:=f_{s} 1_{s, T]}(t)$. This shows that $\xi$ is a $\left(\nu,{ }^{\nu} \mathcal{G}\right)$-martingale, and we conclude by an application of Lévy theorem together with Girsanov theorem; indeed, the quadratic variation of $\xi$ at $t$ must be $t$, by the assumption $\nu \ll \gamma$.

Finally, with the aid of (3.4), we see that the transport plan $\hat{\pi}:=(\xi, i d)_{\#} \nu$ is optimal for (3.2). Moreover, since $\bar{\omega}$ is continuous, the uniqueness of the semimartingale decomposition entails that the process $A$ found in the proof of $(i i) \Rightarrow(i)$ must have not depended on which $\pi$ (with finite cost in (3.2)) we started with. 
In the setting of Theorem 3.1, we note that Lemma 5.5 and Theorem 5.6 stated in Section 5 apply, since $\gamma$ satisfies the weak continuity property (5.2), and the total variation of the difference of the coordinate processes is bounded from below and lower semicontinuous w.r.t. supremum norm. Therefore, $\Pi^{\mathcal{F}, \mathcal{G}}(\gamma, \nu)$ is weakly compact, the problem in (3.2) is attained, and furthermore duality holds.

We now state the main theoretical result of the article, that provides a necessary and sufficient condition for a Brownian motion to remain a semimartingale in an enlarged filtration. We use the notations introduced in Section 2.2.

Theorem 3.2 (Semimartingale preservation property). The following are equivalent:

(i) any process $B$, on some probability space $(\Omega, \mathcal{H}, \mathbb{P})$, which is a Brownian motion in its natural filtration $\mathcal{F}^{B}$, remains a semimartingale in the enlarged filtration $\mathcal{G}^{B}$;

(ii) the causal transport problem (3.2) is finite for some measure $\nu \sim \gamma$.

Moreover, when (i)-(ii) hold, and denoting by $B=\widetilde{B}+N$ the semimartingale decomposition of $B$ in $\mathcal{G}^{B}$, we have that $(\widetilde{B}, B)$ is a causal coupling with respect to $\mathcal{F}^{\tilde{B}}$ and $\mathcal{G}^{B}$.

When (i)-(ii) hold, the idea is that $(\widetilde{B}, B)$ is an optimal coupling (possibly under a different measure) for a causal transport problem as in (3.2). Note that the characterization of semimartingale preservation is given in terms of finiteness of the causal transport problem (3.2) and does not depend on its actual value.

Proof. $(i i) \Rightarrow(i)$ : By Theorem 3.1, there exists a continuous, ${ }^{\nu} \mathcal{G}$-adapted, integrable variation process $A$ such that the process $\xi_{t}(\bar{\omega}):=\bar{\omega}_{t}-A_{t}(\bar{\omega})$ is a $(\nu, \mathcal{G})$-Brownian motion. Since $\nu \sim \gamma$, Girsanov theorem implies that $\bar{\omega}$ is a $(\gamma, \mathcal{G})$-semimartingale. Moreover, since $\bar{\omega}$ is the coordinate process on $\mathcal{C}$, and from $B_{\#} \mathbb{P}=\gamma$ and $\mathcal{G}^{B}=B^{-1}(\mathcal{G})$, we have that $B$ is a $\left(\mathbb{P}, \mathcal{G}^{B}\right)$-semimartingale.

$(i) \Rightarrow(i i)$ : Let $B=M+U$ be the semimartingale decomposition of $B$ in $\mathcal{G}^{B}$, with $M$ a $\left(\mathbb{P}, \mathcal{G}^{B}\right)$-Brownian motion and $U$ a finite variation process, so that in particular $V_{T}(U)<\infty$. Since $0<\left(1+V_{T}(U)\right)^{-1} \leq 1$, we have

$$
c^{-1}:=\mathbb{E}^{\mathbb{P}}\left[\left(1+V_{T}(U)\right)^{-1}\right] \in(0,1],
$$

and $Z_{T}:=c\left(1+V_{T}(U)\right)^{-1}>0 \mathbb{P}$-a.s., with $\mathbb{E}^{\mathbb{P}}\left[Z_{T}\right]=1$. We can then define a probability measure $\mathbb{Q}$ on $\left(\Omega, \mathcal{F}_{T}^{B}\right)$ via $\frac{d \mathbb{Q}}{d \mathbb{P}}:=Z_{T}$, so that $\mathbb{Q} \sim \mathbb{P}$ and

$$
\mathbb{E}^{\mathbb{Q}}\left[V_{T}(U)\right]=c \mathbb{E}^{\mathbb{P}}\left[\frac{V_{T}(U)}{1+V_{T}(U)}\right] \leq c<\infty .
$$

Let $Z$ be the $\left(\mathbb{P}, \mathcal{G}^{B}\right)$-martingale defined from $Z_{T}$. Then, by Girsanov theorem, $B$ has decomposition

$$
B_{t}=\widetilde{M}_{t}+\left(\int_{0}^{t} \frac{d\langle Z, M\rangle_{s}}{Z_{s}}+U_{t}\right),
$$

where $\widetilde{M}:=M-\int_{0} \cdot \frac{d\langle Z, M\rangle_{s}}{Z_{s}}$ is a $\left(\mathbb{Q}, \mathcal{G}^{B}\right)$-Brownian motion. Moreover,

$$
\begin{aligned}
\mathbb{E}^{\mathbb{Q}}\left[\int_{0}^{T}\left|\frac{d\langle Z, M\rangle_{s}}{Z_{s}}\right|\right] & =\mathbb{E}^{\mathbb{P}}\left[\int_{0}^{T}\left|d\langle Z, M\rangle_{s}\right|\right] \leq \mathbb{E}^{\mathbb{P}}\left[\sqrt{\langle Z, Z\rangle_{T}} \sqrt{\langle M, M\rangle_{T}}\right] \\
& =\sqrt{T} \mathbb{E}^{\mathbb{P}}\left[\sqrt{\langle Z, Z\rangle_{T}}\right] \leq K \sqrt{T} \mathbb{E}^{\mathbb{P}}\left[\sup _{t \in[0, T]} Z_{t}\right] \leq c K \sqrt{T}
\end{aligned}
$$

by the Kunita-Watanabe inequality and the Burkholder-Davis-Gundy inequality (with constant $K)$. Together with (3.5), this implies that the process $N:=\int_{0}^{\cdot} \frac{d\langle Z, M\rangle_{s}}{Z_{s}}+U$ is of $\mathbb{Q}$-integrable variation. This shows that $(i i)$ holds with $\nu:=B_{\#} \mathbb{Q}\left(\gamma=B_{\#} \mathbb{P}\right.$ and $\mathbb{Q} \sim \mathbb{P}$ imply $\left.\nu \sim \gamma\right)$, as $\pi=(\widetilde{M}, B)_{\#} \mathbb{Q} \in \Pi^{\mathcal{F}, \mathcal{G}}(\gamma, \nu)$ has finite cost in (3.2).

Finally, by Lemma $2.6,(\widetilde{B}, B)$ is a causal coupling with respect to $\mathcal{F}^{\tilde{B}}$ and $\mathcal{G}^{B}$. 
From the above results it is clear that if there is one causal transport in $\Pi^{\mathcal{F}, \mathcal{G}}(\gamma, \nu)$, for some measure $\nu \sim \gamma$, for which the difference of the coordinates is of integrable variation and a.s. absolutely continuous, then the finite variation part in the semimartingale decomposition of the Brownian motion in the enlarged filtration is also absolutely continuous, see Section 3.2.

Remark 3.3 (Multidimensional processes). We want to point out that the previous theorems can be easily extended to a multidimensional setting. Indeed, instead of the path space $\mathcal{C}$, that accommodates 1-dimensional continuous processes, we can consider $\mathcal{C}^{d}$, path space for d-dimensional continuous processes. In this case, we write $\left\{\left(a_{t}^{i}\right)_{t \in[0, T]}\right\}_{i=1}^{d} \mapsto V_{T}(a):=\sum_{i=1}^{d} V_{T}\left(a^{i}\right)$ in (3.1)(3.2) for the variation of a multidimensional process. Then the proof of Theorem 3.1 follows exactly the same arguments, where the dual projections are now taken componentwise. As for Theorem 3.2, one should define $Z_{T}$ as $c /\left(1+\sum_{i=1}^{d} V_{T}\left(U_{i}\right)\right)$ instead.

\subsection{The absolutely continuous case}

In many well-known filtration enlargements, the finite variation part in the semimartingale decomposition of the Brownian motion in the enlarged filtration is absolutely continuous (as in the examples (2.4), (2.5) and (2.6) above), i.e. is of the form

$$
d B_{t}=d \widetilde{B}_{t}+b_{t}(B) d t
$$

This is true, for example, in the case of initial enlargement of filtrations under Jacod's assumption (see [Jac85], and Section 3.3 below) and under Yor's method (see [Yor97, Sect. 12.1]), as well as in the case of progressive enlargement with a random time (see [JY78] and [Jeu80]); for general enlargements see [ADI06]. That is why this is a framework of major interest which deserves a deeper analysis. In analogy to Theorems 3.1 and 3.2, we can give necessary and sufficient conditions for such a decomposition to hold, together with a characterization of $b$ in terms of causal transport. We will use the notation $(\widehat{\overline{\omega-\omega}})$ to indicate the density of the process $\bar{\omega}-\omega$ when it exists, that is,

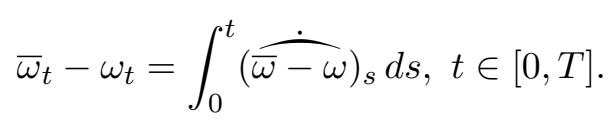

Theorem 3.4. Let $\nu$ be some measure on $\mathcal{C}$ such that $\nu \ll \gamma$, and let $\rho: \mathbb{R} \rightarrow \mathbb{R}_{+}$be a convex even function such that $\rho(+\infty)=+\infty$ and $\rho(0)=0$. Then the following are equivalent:

(i) for some $\mathcal{G}$-predictable process $\alpha=\alpha(\bar{\omega})$ such that

$$
\mathbb{E}^{\nu}\left[\int_{0}^{T} \rho\left(\alpha_{s}\right) d s\right]<\infty
$$

the process $\xi_{t}(\bar{\omega}):=\bar{\omega}_{t}-\int_{0}^{t} \alpha_{s}(\bar{\omega}) d s$ is a $\left(\nu,{ }^{\nu} \mathcal{G}\right)$-Brownian motion;

(ii) the following causal optimal transport problem is finite:

$$
\inf _{\pi \in \Pi^{\mathcal{F}, \mathcal{G}}(\gamma, \nu)} \mathbb{E}^{\pi}\left[\int_{0}^{T} \rho\left((\widehat{\overline{\omega-\omega}})_{t}\right) d t\right]
$$

Moreover, whenever (i)-(ii) hold, then $\hat{\pi}:=(\xi, i d)_{\#} \nu$ belongs to $\Pi^{\mathcal{F}, \mathcal{G}}(\gamma, \nu)$, it is optimal for (3.6), and for every $\pi \in \Pi^{\mathcal{F}, \mathcal{G}}(\gamma, \nu)$ with finite cost in (3.6), it holds that the process $\tilde{\alpha}(\omega, \bar{\omega}):=$ $\alpha(\bar{\omega})$ equals the predictable projection of $(\widetilde{\overline{\omega-\omega}})$ with respect to $\left(\pi,\{\emptyset, \mathcal{C}\} \otimes^{\nu} \mathcal{G}\right)$.

Theorem 3.5 (Semimartingale preservation property). The following are equivalent: 
(i) any process $B$, on some probability space $(\Omega, \mathcal{H}, \mathbb{P})$, which is a Brownian motion in its natural filtration $\mathcal{F}^{B}$, remains a semimartingale in the enlarged filtration $\mathcal{G}^{B}$, with decomposition

$$
d B_{t}=d \widetilde{B}_{t}+b_{t}(B) d t
$$

(ii) the causal transport problem (3.6) is finite for some measure $\nu \sim \gamma$ and some function $\rho$ as in Theorem 3.4.

Moreover, if (ii) holds for $\nu=\gamma$, then the value of the causal transport problem (3.6) equals $\mathbb{E}^{\gamma}\left[\int_{0}^{T} \rho\left(b_{t}(B)\right) d t\right]<\infty$, hence the information drift in (3.7) is $\rho$-integrable.

Remark 3.6. For $\rho(x)=x^{2} / 2$, the cost in (3.6) is called Cameron-Martin cost. In this case, finiteness of problem (3.6) for $\nu=\gamma$ is equivalent to square integrability of the drift in (3.7), by Theorem 3.5. When this holds, one can apply Girsanov theorem, which ensures that $B$ is a Brownian motion in $\mathcal{G}^{B}$ under a change of measure. Therefore, by martingale representation, the $H^{\prime}$-hypothesis between $\mathcal{F}^{B}$ and $\mathcal{G}^{B}$ follows, i.e. all $\mathcal{F}^{B}$-semimartingales remain semimartingales w.r.t. $\mathcal{G}^{B}$. Square integrability of the drift holds for example when initially enlarging with a discrete random variable as in case (1) of Section 2.2.1 when the variable takes finitely many values, while it fails in the Brownian bridge case and for progressive enlargements with last hitting times, as in (2) and (3) of Section 2.2.1. We stress the fact that the semimartingale property being preserved by the Brownian motion is usually not enough to guarantee the $H^{\prime}$ hypothesis, see [JY79]. In the case of initial enlargements with a random variable, we also have that the value of the causal problem (3.6) equals the mutual information between the Brownian motion $B$ and such random variable, see Section 3.3.

The proofs of the above theorems follow the same steps of the proofs of Theorems 3.1 and 3.2 , so we omit them. One simply observes that $\rho(x) \geq m|x|+n$ for some $m>0$, so the relevant processes in the proofs are of integrable and a.s. absolutely continuous variation. Then one recalls that for an integrable variation process $X$ which is absolutely continuous, say $X=\int_{0} a_{t} d t$, the dual predictable projection of $X$ w.r.t. some filtration $\mathcal{H}$ is also absolutely continuous, and is indistinguishable from both $\int_{0}^{\cdot p} a_{t} d t$ and $\int_{0}^{\cdot o} a_{t} d t$, where ${ }^{p} a$ and ${ }^{o} a$ are the predictable and optional projections of $a$ w.r.t. $\mathcal{H}$, respectively.

Remark 3.7. In order to establish an analogue of Theorems 3.2 and 3.5 for general continuous semimartingales, one needs to consider the following condition for transport plans instead of causality:

$$
\mathbb{E}^{\pi}\left[\left(\omega_{t}-\omega_{s}\right) f_{s}(\bar{\omega})\right]=0 \quad \forall 0 \leq s<t \leq T, f_{s} \in L^{\infty}\left(\mathcal{C},{ }^{\nu} \mathcal{G}_{s}, \nu\right) .
$$

In the present paper we prefer to work with the original concept of causality rather than with (3.8). For this reason, we only work in a Brownian framework.

The proofs sketched before the remark are of stochastic analysis flavour, exactly as for Theorems 3.1 and 3.2. We now describe what the optimal transport perspective has to say in the absolutely continuous case. An interesting observation is that in this setting we can actually say more about the problem dual to (3.6), which, as Theorem 5.6 below points out, is given by

$$
\sup _{\substack{\psi \in C_{b}(\mathcal{C}), h \in \mathfrak{H} \\ \psi(\bar{\omega}) \leq \int_{0}^{T} \rho\left((\overline{\bar{\omega}-\omega})_{t}\right) d t+h(\omega, \bar{\omega})}} \mathbb{E}^{\nu}[\psi],
$$


where ${ }^{1}$

$$
\mathfrak{H}:=\operatorname{span}\left(\left\{g(\bar{\omega})\left[f(\omega)-\mathbb{E}^{\gamma}\left[f \mid \mathcal{F}_{t}\right](\omega)\right]: f \in C_{b}(\mathcal{C}), g \in B_{b}\left(\mathcal{C}, \mathcal{G}_{t}\right), t \in[0, T]\right\}\right) .
$$

Observe that $\gamma$ is implicitly present in Problem (3.9), since it appears in the constraints set $\mathfrak{H}$. The next result is proved in Appendix B, where its ingredients are more closely examined. The necessary elementary facts on Orlicz spaces are given in Appendix A. We stress that even without anticipation of information (i.e. $\mathcal{F}=\mathcal{G}$ ) this is a novel result.

We will talk of refined dual problem, by referring to the following formulation:

$$
\sup \left\{\mathbb{E}^{\nu}\left[\int_{0}^{T} F_{t}(\bar{\omega}) d \bar{\omega}_{t}-\int_{0}^{T} \rho^{*}\left(F_{t}(\bar{\omega})\right) d t\right]: F \in S_{a}(\mathcal{G})\right\},
$$

where $\rho^{*}$ denotes the convex conjugate of $\rho$, and $S_{a}(\mathcal{G})$ denotes the set of simple previsible processes w.r.t. $\mathcal{G}$, namely,

$S_{a}(\mathcal{G}):=\left\{\sum_{i=1}^{m} F^{i}(\bar{\omega}) \mathbb{I}_{\left(\tau_{i}, \tau_{i+1}\right]}(t): m \in \mathbb{N}, 0 \leq \tau_{1} \leq \cdots \leq \tau_{m} \leq T \mathcal{G}\right.$-stopping times, $\left.F^{i} \in B_{b}\left(\mathcal{G}_{\tau_{i}}\right)\right\}$,

so the first integral in (3.10) is defined as a finite sum as customary. Moreover, we write $\frac{\rho(0)}{0}$ for $\lim _{x \searrow 0} \frac{\rho(x)}{x}$, and $\frac{\rho(+\infty)}{+\infty}$ for $\lim _{x \nearrow+\infty} \frac{\rho(x)}{x}$.

Theorem 3.8. Let $\nu \ll \gamma$, and suppose that $\rho$ is strictly convex, even, and satisfies for some $C, x_{0}>0$ and $\ell>1$ :

$$
\rho(x)=0 \Leftrightarrow x=0, \frac{\rho(0)}{0}=0, \frac{\rho(+\infty)}{+\infty}=+\infty, \rho(2 x) \leq C \rho(x) \text { and } \rho(x) \leq \frac{\rho(\ell x)}{2 \ell} \text { if } x \geq x_{0} .
$$

Then:

(i) The primal (3.6), the dual (3.9), and the refined dual (3.10), have all the same value.

From now on we assume that this common value is finite.

(ii) The refined dual (3.10) can be computed (without changing its value) over $M^{\rho^{*}}$, the closure of $S_{a}(\mathcal{G})$ w.r.t. the so-called gauge norm

$$
F \mapsto\|F\|_{\rho^{*}}:=\inf \left\{\beta>0: \mathbb{E}^{\nu}\left[\int_{0}^{T} \rho^{*}\left(F_{t} / \beta\right) d t\right] \leq 1\right\},
$$

and it is attained there by a unique optimizer $\hat{F}=\hat{F}(\bar{\omega})$.

(iii) The optimal drift $\alpha$ in Theorem $3.4(i)$ is related to $\hat{F}$ through

$$
\rho^{*}\left(\hat{F}_{t}(\bar{\omega})\right)+\rho\left(\alpha_{t}(\bar{\omega})\right)=\alpha_{t}(\bar{\omega}) \hat{F}_{t}(\bar{\omega}) \quad d \nu \times d t-a . s .
$$

namely $\alpha_{t}(\bar{\omega})=\left(\rho^{*}\right)^{\prime}\left(\hat{F}_{t}(\bar{\omega})\right)$, or equivalently, $\hat{F}_{t}(\bar{\omega}) \in \partial \rho\left(\alpha_{t}(\bar{\omega})\right)$, with $\partial$ denoting sub-differential. (iv) $\xi_{t}(\bar{\omega}):=\bar{\omega}_{t}-\int_{0}^{t} \alpha_{s}(\bar{\omega}) d s$ is a $\left(\nu,{ }^{\nu} \mathcal{G}\right)$-Brownian motion, so if further $\gamma \ll \nu$ we have that the canonical process $\bar{\omega}$ is a $\left(\gamma,{ }^{\nu} \mathcal{G}\right)$-semimartingale.

The refined dual problem has in principle no immediate connection to $\gamma$. In fact, it provides a lower bound for the causal transport problem even if $\nu \nless \gamma$, as we prove in Lemma B.1 in the appendix. It is a non-trivial consequence of Theorem 3.8 that when $\nu \ll \gamma$ the values of the refined dual and the causal transport problem coincide.

The typical examples for which the given conditions on $\rho$ are satisfied, are power functions $\rho(x) \sim|x|^{p}$ with exponent $1<p<+\infty$, which covers the Cameron-Martin case, as well as $\rho(x)=|x|^{a}(1+|\log | x||)$ for $a>1$; see the comments after [RR91, Ch. II.2.3, Corollary 4].

\footnotetext{
${ }^{1}$ Here and thereafter, span denotes the linear space of functions obtained by finite linear combinations of those functions in the generating class.
} 
Remark 3.9. We stress that the dual (3.9) is most often not attained on continuous functions. Still, the refined dual (3.10) admits an optimizer which induces a formal optimal element for (3.9) by setting $h(\omega, \bar{\omega}):=\int_{0}^{T} \hat{F}_{t}(\bar{\omega}) d \omega_{t}$ and

$$
\psi(\bar{\omega}):=\int_{0}^{T} \hat{F}_{t}(\bar{\omega}) d \bar{\omega}_{t}-\int_{0}^{T} \rho^{*}\left(\hat{F}_{t}(\bar{\omega})\right) d t=\int_{0}^{T} \hat{F}_{t}(\bar{\omega}) d \xi_{t}(\bar{\omega})+\int_{0}^{T} \rho\left(\alpha_{t}(\bar{\omega})\right) d t .
$$

Notice that, even though the optimal $\hat{F}$ depends on the cost function $\rho$, the optimal drift $\alpha$ does not. In the Cameron-Martin case $\rho(x)=x^{2} / 2$, we actually get from (3.12) that $\hat{F}=\alpha$ and so $\psi(\bar{\omega})=\int_{0}^{T} \alpha_{t}(\bar{\omega}) d \bar{\omega}_{t}-\int_{0}^{T}\left(\alpha_{t}(\bar{\omega})\right)^{2} / 2 d t$. Furthermore, in the absence of enlargement (i.e. $\mathcal{F}=\mathcal{G})$ we find by Girsanov theorem the identity

$$
\frac{d \nu}{d \gamma}(\bar{\omega})=\exp (\psi(\bar{\omega}))
$$

In words: the causal Kantorovich potential $\psi$ between Wiener measure and $\nu$ is the logarithm of their relative density.

In the proof of Theorem 3.8 (see Appendix B) we extend the stochastic integral $\int_{0}^{T} F_{t}(\bar{\omega}) d \bar{\omega}_{t}$ beyond simple $\mathcal{G}$-previsible integrands via functional analytic arguments, much inspired by [Léo12]. Of course, this could have been done via Theorem 3.4, using that a fortiori the coordinate process $\bar{\omega}$ is a $(\nu, \mathcal{G})$-semimartingale. We avoided this to show that there is a true transport/functional method for this. Likewise, Point $(i v)$ is obtained without using previous results.

Remark 3.10 (Multidimensional processes). As seen in Remark 3.3 for the general case, also the theorems of this section have an analogue in the multidimensional setting. It suffices to define the gauge norm (3.11) as acting on the euclidean norm of $F_{t}(\bar{\omega})$, interpret the r.h.s. of (3.12) as inner product, etc. This is straightforward, so we do not give the details.

\subsection{Initial enlargement: Jacod's condition and connections with the litera- ture}

In this section we analyse the connections between our results with the existing literature on initial enlargement of filtrations. Let $B$ be a Brownian motion on the probability space $\left(\Omega, \mathcal{F}^{B}, \mathbb{P}\right)$. We enlarge the Brownian filtration $\mathcal{F}^{B}$ with a real-valued random variable $L(B)$, and denote by $\mathcal{G}^{B}$ the enlarged filtration, $\mathcal{G}^{B}=\mathcal{F}^{B} \vee \sigma(L(B))$. Let $\ell: \mathcal{F}_{T}^{B} \rightarrow[0,1]$ be the law of $L(B)$, and $\ell_{t}: \Omega \times \mathcal{F}_{T}^{B} \rightarrow[0,1]$ be a regular version of the $\mathcal{F}_{t}^{B}$-conditional law of $L(B), t \in[0, T)$. Jacod's celebrated method [Jac85] relies on the following density hypothesis: for all $t \in[0, T), \ell_{t} \ll \ell$ in the $\mathbb{P}$-a.s. sense. In this case, there exists a suitable version of the density of $\ell_{t}$ with respect to $\ell$, which we denote by $p_{t}^{x}$, such that for every $x \in \mathbb{R}$, the process $\left(p_{t}^{x}\right)_{t \in[0, T)}$ is a martingale on $\left(\Omega, \mathcal{F}^{B}, \mathbb{P}\right)$. Given that we are in a Brownian filtration, this ensures the existence of $\mathcal{F}^{B}$-adapted processes $N^{x}$ such that $p_{t}^{x}=1+\int_{0}^{t} N_{s}^{x} d B_{s}$. The semimartingale decomposition of $B$ in $\mathcal{G}^{B}$ is then given by $B_{t}=\tilde{B}_{t}+A_{t}^{L(B)}$, where the finite variation process is absolutely continuous, that is $A_{t}^{L(B)}=\int_{0}^{t} \alpha_{s}^{L(B)} d s$, with $\alpha_{t}^{x}=N_{t}^{x} / p_{t}^{x}$.

\subsubsection{Relation with Jeulin's characterization}

Proposition 3.8 in [Jeu80] provides necessary and sufficient conditions for a martingale to remain a semimartingale in an initially enlarged filtration, in terms of a change of measure and a 
finiteness condition. The connection with our results can be easily illustrated under Jacod's hypothesis. In case of no change of measure, the finiteness condition in [Jeu80] reads as

$$
\mathbb{E}^{\mathbb{P}}\left[\int_{0}^{T} \int_{\mathbb{R}}\left|N_{t}^{x}\right| \ell(d x) d t\right]<\infty
$$

which ultimately follows by noticing

$$
\mathbb{P}\left(L(B)>x \mid \mathcal{F}_{t}^{B}\right)=\int_{x}^{\infty} \ell_{t}(d a)=\int_{x}^{\infty} p_{t}^{a} \ell(d a)=\int_{x}^{\infty}\left[1+\int_{0}^{t} N_{s}^{a} d B_{s}\right] \ell(d a) .
$$

According to our Theorem 3.5, the causal transport problem (3.6) must be finite for some measure $\nu \sim \gamma$ and some function $\rho$ as in Theorem 3.4. Let $\rho=||,. \nu=\gamma$, and $\hat{\pi}$ be as in Theorem 3.4, that is $\hat{\pi}=(\xi, i d)_{\#} \gamma \in \Pi^{\mathcal{F}, \mathcal{G}}(\gamma, \gamma)$, with $\xi_{t}(\bar{\omega})=\bar{\omega}_{t}-\int_{0}^{t} \alpha_{s}^{L(B)}(\bar{\omega}) d s$. Then

$$
\begin{aligned}
\mathbb{E}^{\hat{\pi}}\left[\int_{0}^{T} \rho\left((\widehat{\overline{\omega-\omega}})_{t}\right) d t\right] & =\mathbb{E}^{\mathbb{P}}\left[\int_{0}^{T}\left|\alpha_{t}^{L(B)}\right| d t\right]=\mathbb{E}^{\mathbb{P}}\left[\int_{0}^{T}\left|\frac{N_{t}^{L(B)}}{p_{t}^{L(B)}}\right| d t\right]=\mathbb{E}^{\mathbb{P}}\left[\int_{0}^{T} \int_{\mathbb{R}}\left|\frac{N_{t}^{x}}{p_{t}^{x}}\right| \ell_{t}(d x) d t\right] \\
& =\mathbb{E}^{\mathbb{P}}\left[\int_{0}^{T} \int_{\mathbb{R}}\left|N_{t}^{x}\right| \ell(d x) d t\right],
\end{aligned}
$$

which is exactly (3.13).

More generally, the change of measure in [Jeu80] plays the role of the measure $\nu$ being possibly different from $\gamma$ in our Theorem 3.5-(ii).

\subsubsection{Reduction to the case of same filtration}

As explained in the "Comparison with Jacod's condition" Section in [ADI07], Jacod's method can be interpreted in the following way: assuming that for almost all $x, \mathbb{P}^{x}:=\mathbb{P}(\cdot \mid L(B)=$ $x) \ll \mathbb{P}$, one applies Girsanov theorem and finds $B-A^{x}$ to be a (local) martingale w.r.t. $\mathbb{P}^{x}$. Then, combining these, one obtains that $B-A^{L(B)}$ is a (local) martingale w.r.t. $\mathbb{P}$ and the enlarged filtration $\mathcal{G}^{B}$. Notably, there is a causal optimal transport counterpart to the method just described. Denote

$$
\gamma^{L=x}:=\text { "conditional law of } B \text { given } L(B)=x " .
$$

Lemma 3.11. Set $\mathcal{G}=\mathcal{F} \vee \sigma(L)$. We have

$$
\inf _{\pi \in \Pi^{\mathcal{F}, \mathcal{G}}(\gamma, \gamma)} \mathbb{E}^{\pi}\left[\int_{0}^{T} \rho\left(\widehat{(\widehat{\overline{\omega-\omega}})_{t}}\right) d t\right]=\int\left\{\inf _{\pi \in \Pi^{\mathcal{F}, \mathcal{F}}\left(\gamma, \gamma^{L=x}\right)} \mathbb{E}^{\pi}\left[\int_{0}^{T} \rho\left((\widehat{\overline{\omega-\omega}})_{t}\right) d t\right]\right\} \ell(d x) .
$$

Observe that the integrand in the r.h.s. of (3.14) is a causal transport problem in itself, but without enlargement of filtration. The proof relies on easily checking that for $\pi \in \Pi^{\mathcal{F}, \mathcal{G}}(\gamma, \gamma)$ one has $\pi^{L(\bar{\omega})=x} \in \Pi^{\mathcal{F}, \mathcal{F}}\left(\gamma, \gamma^{L=x}\right)$, and ultimately on a standard measurable selection argument, and so we omit it.

\subsubsection{Cameron-Martin cost: entropy and mutual information}

In the Cameron-Martin case of $\rho(x)=x^{2} / 2$, [Las15, Lemma 5] implies that the integrand in the r.h.s. of (3.14) equals the relative entropy of $\gamma^{L=x}$ w.r.t. $\gamma$, whenever this is finite. For us this means that

$$
\inf _{\pi \in \Pi^{\mathcal{F}, \mathcal{G}}(\gamma, \gamma)} \mathbb{E}^{\pi}\left[\frac{1}{2} \int_{0}^{T}(\widehat{\overline{\omega-\omega}})_{t}^{2} d t\right]=\int \operatorname{Ent}\left(\gamma^{L=x} \mid \gamma\right) \ell(d x) .
$$

Since the relative entropy $\operatorname{Ent}\left(\gamma^{L=x} \mid \gamma\right)$ is further integrated w.r.t. the law of $L(B)$, we get that the r.h.s. in (3.15) corresponds to the so-called Mutual Information between $B$ and $L(B)$, 
denoted by $\mathrm{I}(B, L(B))$. It is defined as the relative entropy of the joint law $P_{B, L(B)}$ w.r.t. the decoupling measure $P_{B} \otimes P_{L(B)}$, namely:

$$
\mathrm{I}(B, L(B)):=\operatorname{Ent}\left(P_{B, L(B)} \mid P_{B} \otimes P_{L(B)}\right)=\int \operatorname{Ent}\left(\gamma^{L=x} \mid \gamma\right) \ell(d x) .
$$

On the other hand, by Theorem 3.5, the l.h.s. in (3.15) is finite if and only if the information drift $\alpha^{L(B)}$ in the semimartingale decomposition of $B$ w.r.t. $\mathcal{G}^{B}$ is square integrable, in which case the value of the causal transport problem equals $\frac{1}{2} \mathbb{E}^{\gamma}\left[\int_{0}^{T}\left(\alpha_{t}^{L(B)}\right)^{2} d t\right]$. In [PK96] (see also [ADI06], [AIS98]) it is proved that this value corresponds to the additional utility obtained by an investor who maximizes the expected log-utility of terminal wealth in a certain complete market model w.r.t. $\mathcal{G}^{B}$, compared to an investor w.r.t. $\mathcal{F}^{B}$. Further, it is known that this value also equals the relative entropy $\operatorname{Ent}(\mathbb{P} \mid \mathbb{Q})$, where $\mathbb{Q}$ is a probability measure under which $B$ is a $\left(\mathbb{Q}, \mathcal{G}^{B}\right)$-Brownian motion; see Remark 3.6. Putting things together, we have

Corollary 3.12. Assuming that $\gamma^{L=x} \ll \gamma \forall x$ Ł-a.s., then

$$
\inf _{\pi \in \Pi^{\mathcal{F}, \mathcal{G}}(\gamma, \gamma)} \mathbb{E}^{\pi}\left[\frac{1}{2} \int_{0}^{T}(\widehat{\overline{\omega-\omega}})_{t}^{2} d t\right]=\operatorname{Ent}(\mathbb{P} \mid \mathbb{Q})=\int \operatorname{Ent}\left(\gamma^{L=x} \mid \gamma\right) l(d x)=I(B, L(B))
$$

Note that the equality with the mutual information recovers the result of [ADI06, Theorem 5.13] using our methods. If the initial enlargement is done by a discrete random variable as in Section 2.2.1-(1), then

$$
\inf _{\pi \in \Pi^{\mathcal{F}, \mathcal{G}}(\gamma, \gamma)} \mathbb{E}^{\pi}\left[\frac{1}{2} \int_{0}^{T}(\widehat{\overline{\omega-\omega}})_{t}^{2} d t\right]=-\sum_{n} p_{n} \ln \left(p_{n}\right)
$$

where $p_{n}=\mathbb{P}\left(C_{n}\right)$, and the term on the r.h.s. is referred to as the entropy of the partition $\left\{C_{n}\right\}_{n \in \mathbb{N}}$; see [Yor85] and [AIS98].

\section{Robust transport bounds for stochastic optimization}

In this section we show how the causal transport framework allows us to give robust estimates for the value of additional information, as well as model sensitivity, for some classical stochastic optimization problems in continuous-time. By value of information we mean the difference between the optimal values of these problems with and without additional information (i.e. w.r.t. the enlarged and the original filtration, respectively). By model sensitivity we mean the difference between the optimal values of these problems under two different probabilistic models (i.e. reference probability measures). For the value of information, the main idea is to take "causal projections" of candidate optimizers in the problem with the larger filtration, so building a feasible element in the problem with the smaller filtration, and making a comparison possible. For model sensitivity, it means to project an optimizer under one model in order to build a feasible element for the other model, which enables a direct comparison. In discrete-time and in the setting of model sensitivity in multistage stochastic programming, this idea goes back to [Pfl09, PP12].

We start with Section 4.1, on optimal stopping problems, for which the outlined projection approach is more delicate and fully novel to the best of our knowledge (even in discrete-time). Then in Section 4.2 we deal with utility maximization with portfolio constraints; this is a prominent example of a controlled linear system, and indeed the same arguments would be applicable to such systems in general. In both optimization problems considered below, we will obtain robust estimates in terms of causal minimization problems. These estimates not 
only provide a theoretical insight, they also give us bounds which can be actually obtained or approximated in several situations of interest. We refer the reader to the work [BNT19], where an $L^{2}$ causal transport problem is solved in the case where the marginals are diffusion processes and the filtrations are the canonical ones. The authors characterize the value of the problem via PDE techniques. Thus in the case of optimal stopping (see Proposition 4.4 below) our upper bound could be obtained/approximated by high-dimensional but otherwise standard PDEs; this has to be compared with the difficulty of solving the free boundary problems associated to optimal stopping. Another promising direction regarding the computation of our bounds, is the discretization method introduced in [ABC18]; we are currently working on the full numerical implementation of this method.

\subsection{Optimal stopping}

Here we consider the framework of Section 2.2, with canonical space $\mathcal{C}:=\mathcal{C}[0, T]$ and filtrations $\mathcal{F} \subseteq \mathcal{G}$ on it. We begin with the definition of a randomized stopping time:

Definition 4.1. A randomized stopping time $\Sigma$ with respect to a filtration $\mathcal{H}$ and a probability measure $\mu \in \mathcal{P}(\mathcal{C})$, written $\Sigma \in R S T(\mathcal{H}, \mu)$, is an increasing right-continuous $\mathcal{H}$-adapted process on $[0, T]$, with $\Sigma_{0}=0$ and $\Sigma_{T}=1, \mu-$ a.s.

This notion generalizes the concept of stopping time, say $\tau$, according to which a path $\omega$ is stopped at a unique point in time $\tau(\omega)$. Stopping according to a randomized stopping time $\Sigma$ means that a path $\omega$ is stopped in $[0, t]$ with probability $\Sigma_{t}(\omega)$. We recommend [BCH17, Sect. 3.2] for a modern view on this matter, and refer to [BC77] for the original motivation/definition.

The next lemma is of fundamental importance for our applications. It identifies what causal dual optional projections do to randomized stopping times:

Lemma 4.2. Let $\Sigma \in R S T(\mathcal{G}, \nu)$. Then, for any $\mu \in \mathcal{P}(\mathcal{C})$ and any causal transport plan $\pi \in \Pi^{\mathcal{F}, \mathcal{G}}(\mu, \nu)$, there is a randomized stopping time $\tilde{\Sigma} \in R S T(\mathcal{F}, \mu)$ such that

$$
\mathbb{E}^{\pi}\left[\int_{0}^{T} \ell(\omega, t) d \Sigma_{t}(\bar{\omega})\right]=\mathbb{E}^{\mu}\left[\int_{0}^{T} \ell(\omega, t) d \tilde{\Sigma}_{t}(\omega)\right]
$$

for all $\mathcal{F}$-optional processes $(\omega, t) \mapsto \ell(\omega, t)$ which are bounded or positive.

Proof. Let $\tilde{\Sigma}(\omega, \bar{\omega})$ be the dual optional projection of $\Sigma=\Sigma(\bar{\omega})$ with respect to $\left(\pi,{ }^{\pi}(\mathcal{F} \otimes\{\emptyset, \mathcal{C}\})\right)$. From Lemma C.1, we may assume that $\tilde{\Sigma}(\omega, \bar{\omega})=\tilde{\Sigma}(\omega)$, and from Lemma C.2 below we have that $\tilde{\Sigma}$ equals the optional projection of $\Sigma$ with respect to $\left(\pi,{ }^{\pi}(\mathcal{F} \otimes\{\emptyset, \mathcal{C}\})\right)$. Moreover, by [DM80, Lemma 7, App. I], we can assume $\tilde{\Sigma}$ to be $(\mathcal{F} \otimes\{\emptyset, \mathcal{C}\})$-optional. This implies that $\tilde{\Sigma}$ lies in the interval $[0,1]$ too, and hence belongs to $\operatorname{RST}(\mathcal{F}, \mu)$.

Our purpose is to quantitatively gauge, via causal transport arguments, the dependence of optimal stopping problems on $[0, T]$ with respect to the filtration or the reference probability measure. See [LP90, CT07] or the seminal but unpublished work [Ald81], for the related issue of (qualitative) stability of these problems. Lemma 4.2 above suggests that we should rather define optimal stopping over randomized stopping times. It is well-known that, in the non-anticipative case, one can move between formulations over stopping times and over randomized stopping times. That this is also true in the anticipative case is somewhat hidden in the aforementioned articles, so we sketch the arguments for convenience of the reader: 
Lemma 4.3. Let $\nu \in \mathcal{P}(\mathcal{C})$, and let $(\omega, t) \mapsto \ell(\omega, t)$ be measurable, $\mathcal{F}$-optional, bounded or positive. Then

$$
\inf \left\{\mathbb{E}^{\nu}\left[\ell_{\tau}\right]: \tau \text { a } \mathcal{G} \text {-stopping time on } \mathcal{C}, \tau \leq T\right\}=\inf _{\Sigma \in R S T(\mathcal{G}, \nu)} \mathbb{E}^{\nu}\left[\int \ell_{t} d \Sigma_{t}\right]
$$

Furthermore, let $(\Omega, \mathcal{H}, \mathbb{P})$ be a complete filtered probability space, $X: \Omega \rightarrow \mathcal{C}$ measurable and $\mathcal{H}$-adapted with $X_{\#} \mathbb{P}=\nu$. Assuming that

$$
\forall t \leq T, X^{-1}\left(\mathcal{G}_{T}\right) \text { is conditionally independent of } \mathcal{H}_{t} \text { given } X^{-1}\left(\mathcal{G}_{t}\right),
$$

we further have that the common value in (4.1) equals

$$
\inf \left\{\mathbb{E}^{\mathbb{P}}[\ell(X, \tau)]: \tau \text { a } \mathcal{H} \vee X^{-1}(\mathcal{G}) \text {-stopping time on } \Omega, \tau \leq T\right\} .
$$

Proof. We first prove (4.1), following [CT07, Proof of Lemma 9]. Evidently the r.h.s. in (4.1) is the lesser one. For the converse inequality, take $\Sigma \in R S T(\mathcal{G}, \nu)$ and define

$$
(\omega, x) \in \mathcal{C} \times[0,1] \mapsto \tau(\omega, x):=\inf \left\{t \in[0, T]: \Sigma_{t}>x\right\},
$$

so by [DM80, Ch. VI.55] we have

$$
\mathbb{E}^{\nu}\left[\int_{0}^{T} \ell(\omega, t) d \Sigma_{t}(\omega)\right]=\mathbb{E}^{\nu}\left[\int_{0}^{1} \ell(\omega, \tau(\omega, x)) d x\right] .
$$

Observe that for $x$ fixed and each $t$ we have $\{\omega \in \mathcal{C}: \tau(\omega, x)>t\}=\left\{\omega \in \mathcal{C}: \Sigma_{t} \leq x\right\} \in \mathcal{G}_{t}$, hence $\omega \mapsto \tau(\omega, x)$ is a $\mathcal{G}$-stopping time on $\mathcal{C}$. Applying Fubini-Tonelli theorem, we find

$$
\mathbb{E}^{\nu}\left[\int \ell(\omega, t) d \Sigma_{t}(\omega)\right]=\int_{0}^{1} \mathbb{E}^{\nu}[\ell(\omega, \tau(\omega, x))] d x,
$$

and since the integrand in the r.h.s. here is for each $x$ larger than the l.h.s. of (4.1), this establishes the equality. As for (4.3), one follows the arguments in [LP90, Proposition 3.5], or more precisely their extension in [CT07, Lemma 17].

If $\nu$ above is Markov (resp. Wiener) and $\mathcal{F}=\mathcal{G}$, then (4.2) is equivalent to $X$ being Markov (resp. Brownian motion) w.r.t. $\mathcal{H}$. This should convey the message that both Condition (4.2) and Problem (4.3) are natural in our more general context.

We now look at optimal stopping under $(\mathcal{F}, \mu)$, which by the previous lemma equals

$$
v^{\mathcal{F}, \mu}:=\inf _{\Sigma \in R S T(\mathcal{F}, \mu)} \mathbb{E}^{\mu}\left[\int \ell_{t} d \Sigma_{t}\right]
$$

We want to compare this problem with the one where extra information (anticipation) is available and/or the law of the process to be stopped is different, namely (again by Lemma 4.3)

$$
v^{\mathcal{G}, \nu}:=\inf _{\Sigma \in R S T(\mathcal{G}, \nu)} \mathbb{E}^{\nu}\left[\int \ell_{t} d \Sigma_{t}\right]
$$

The comparison of $v^{\mathcal{F}, \mu}$ with $v^{\mathcal{G}, \mu}$ corresponds to assessing the cost of information/anticipation. On the other hand, the comparison of $v^{\mathcal{F}, \mu}$ with $v^{\mathcal{F}, \nu}$ corresponds to the study of the dependence of non-anticipating optimal stopping with respect to different reference measures, or equivalently, with respect to different processes; in other words, model sensitivity. We have: 
Proposition 4.4. Assume that $v^{\mathcal{F}, \mu}$ and $v^{\mathcal{G}, \nu}$ are both finite, and that the cost function $\ell: \mathcal{C} \times \mathbb{R}_{+}$ is optional and $K$-Lipschitz in its first argument with respect to a metric $d$ on $\mathcal{C}$, uniformly in time (i.e. in the second argument). Then we have

$$
v^{\mathcal{F}, \mu}-v^{\mathcal{G}, \nu} \leq K \inf _{\pi \in \Pi^{\mathcal{F}, \mathcal{G}}(\mu, \nu)} \mathbb{E}^{\pi}[d(\omega, \bar{\omega})]
$$

In particular, in the two special cases of interest we have:

(i) If $\mu=\nu$, then

$$
0 \leq v^{\mathcal{F}, \mu}-v^{\mathcal{G}, \mu} \leq K \inf _{\pi \in \Pi^{\mathcal{F}, \mathcal{G}}(\mu, \mu)} \mathbb{E}^{\pi}[d(\omega, \bar{\omega})]
$$

(ii) If $\mathcal{F}=\mathcal{G}$, then

$$
\left|v^{\mathcal{F}, \mu}-v^{\mathcal{F}, \nu}\right| \leq K \inf _{\pi \in \Pi_{b c}^{\mathcal{F}, \mathcal{F}}(\mu, \nu)} \mathbb{E}^{\pi}[d(\omega, \bar{\omega})],
$$

where the constraint in the transport problem in the right-hand side of (4.6) means that both $\pi \in \Pi^{\mathcal{F}, \mathcal{F}}(\mu, \nu)$ and $\tilde{\pi} \in \Pi^{\mathcal{F}, \mathcal{F}}(\nu, \mu)$, where $\tilde{\pi}=((\omega, \bar{\omega}) \mapsto(\bar{\omega}, \omega))_{\#} \pi$.

Proof. Take an optimizer $\Sigma$ for $(O S(\mathcal{G}, \nu))$ (same argument holds for an optimizing sequence). We write this in the $\bar{\omega}$-variable and consider any causal transport $\pi$ between $\mu$ and $\nu$. Let $\tilde{\Sigma} \in R S T(\mathcal{F}, \mu)$ be the randomized stopping time associated to $\Sigma$, as in Lemma 4.2. We have

$$
v^{\mathcal{F}, \mu} \leq \mathbb{E}^{\mu}\left[\int_{0}^{T} \ell(\omega, t) d \tilde{\Sigma}_{t}(\omega)\right]=\mathbb{E}^{\pi}\left[\int_{0}^{T} \ell(\omega, t) d \Sigma_{t}(\bar{\omega})\right] .
$$

Hence the difference can be bounded above as follows

$$
v^{\mathcal{F}, \mu}-v^{\mathcal{G}, \nu} \leq \mathbb{E}^{\pi}\left[\int_{0}^{T}[\ell(\omega, t)-\ell(\bar{\omega}, t)] d \Sigma_{t}(\bar{\omega})\right] \leq K T \mathbb{E}^{\pi}[d(\omega, \bar{\omega})] .
$$

Being $\pi$ a generic causal transport between the measures $\mu$ and $\nu$, we get the bound in (4.4).

In the case (i), obviously $v^{\mathcal{F}, \mu} \geq v^{\mathcal{G}, \mu}$, since only the set of feasible optimization variables changes. As for the case (ii), exchanging the roles of $\mu$ and $\nu$ we get

$$
\left|v^{\mathcal{F}, \mu}-v^{\mathcal{F}, \nu}\right| \leq K \max \left\{\inf _{\pi \in \Pi^{\mathcal{F}, \mathcal{F}}(\mu, \nu)} \mathbb{E}^{\pi}[d(\omega, \bar{\omega})], \inf _{\pi \in \Pi^{\mathcal{F}, \mathcal{F}}(\nu, \mu)} \mathbb{E}^{\pi}[d(\omega, \bar{\omega})]\right\} \leq K \inf _{\pi \in \Pi_{b c}^{\mathcal{F}, \mathcal{F}}(\mu, \nu)} \mathbb{E}^{\pi}[d(\omega, \bar{\omega})] .
$$

The last inequality follows since the cost $d$ is symmetric (as a metric), implying that the r.h.s. can be computed on $\Pi_{b c}^{\mathcal{F}, \mathcal{F}}(\mu, \nu)$ or $\Pi_{b c}^{\mathcal{F}, \mathcal{F}}(\nu, \mu)$ equivalently.

Replacing the Lipschitz condition in Proposition 4.4 by uniform continuity, one obtains analogue results involving a modulus of continuity. Notice that if $\ell(x, t)=f\left(\int_{0}^{t} x_{s} d s\right)$ and $f$ is Lipschitz, then Proposition 4.4 applies with $d(\omega, \bar{\omega})=\|\omega-\bar{\omega}\|_{L^{2}[0, T]}$. The corresponding optimal stopping problem can be interpreted e.g. as the optimal exercise of an American option of Asian type. In this case the PDE method of [BNT19] can be applied in order to characterize and compute the upper bound in case $\mathcal{F}=\mathcal{G}$. On the other hand, if $\ell(x, t)=f\left(x_{t}\right)$ and $\ell(x, t)=f\left(\sup _{s \leq t} x_{s}\right)$, then again the assumptions of Proposition 4.4 are satisfied, with $d(\omega, \bar{\omega})=$ $\|\omega-\bar{\omega}\|_{\infty}$. In this case the bound in (4.4) can be further majorized up to a multiplicative constant by

$$
\inf _{\pi \in \Pi^{\mathcal{F}, \mathcal{G}}(\mu, \nu)} \mathbb{E}^{\pi}\left[V_{T}(\bar{\omega}-\omega)\right]
$$

Remark 4.5. Note that, for any choice of filtrations $\mathcal{F}$ and $\mathcal{G}$ on $\mathcal{C}$ (non-necessarily satisfying $\mathcal{F} \subseteq \mathcal{G})$, Lemma 4.2 still holds true. This means that, under the assumptions of Proposition 4.4, the bound in (4.4) still holds, thus giving an estimate of the difference between the optimal stopping problems of agents with different information (non-necessarily one bigger than the other). 


\subsection{Utility maximization}

This part follows in spirit the previous section. We want to compare the optimal value of expected utility from terminal wealth, over a fixed finite time horizon $[0, T]$, when the reference filtration is enlarged by anticipation of information in the sense of Section 2.2. A wide literature is devoted to the utility maximization problem, in complete or incomplete markets, and with or without additional constraints; see [XS92, KLSX91, KLS87, CK92] among the earliest articles on the subject. Pikovsky and Karatzas [PK96] were the first ones to include anticipation of information. In a complete market, and for initial filtration enlargements, they provide the explicit value of this anticipation of information, in terms of log-utility maximization, with or without short-selling constraints; see also [AIS98, ADI06].

In this section we consider possibly incomplete markets, and any kind of anticipation of information (not just initial), and give an estimate of the value of information in terms of utility maximization under short-selling constraints, for a class of utility functions which includes the logarithm among other well-known ones; see Assumption 4.7. In order to do this, we set $\mathcal{X}=\mathcal{Y}=\mathcal{C}^{d}=\mathcal{C}\left([0, T], \mathbb{R}^{d}\right)$, the space of continuous $\mathbb{R}^{d}$-valued functions on the interval $[0, T]$, and keep the notation $\omega, \bar{\omega}$ for the coordinate processes. Let $(\Omega, \mathbb{P})$ be a probability space, equipped with $\mathcal{F}^{B}$, the natural filtration of a $d$-dimensional Brownian motion $B=\left(B_{1}, \ldots, B_{d}\right)^{*}$, i.e. $\mathcal{F}^{B}=\sigma\left(B_{1}, \ldots, B_{d}\right)$, augmented so as to satisfy the usual conditions. We use notation analogous to that of Section 2.2, and denote by $\mathcal{G}^{B}$ the enlargement of the filtration $\mathcal{F}^{B}$ with some anticipation of information on the evolution of $B$. Hence $\mathcal{G}^{B}$ represents the information available to the informed agent. In this section we assume:

Assumption 4.6. The process $B$ remains a semimartingale with respect to $\mathcal{G}^{B}$, say with semimartingale decomposition $B=\widetilde{B}+A$.

We consider a financial market consisting of a riskless asset (bond), which we normalize to 1 , and $m \leq d$ risky assets whose price dynamics are described by the stochastic equations

$$
d S_{t}^{i}=S_{t}^{i}\left(b_{t}^{i} d t+\sum_{j=1}^{d} \sigma_{t}^{i, j} d B_{t}^{j}\right), \quad i=1, \ldots, m,
$$

with initial condition $S_{0}^{i}=s_{0}^{i}>0$. The vector process $b=\left(b_{t}^{1}, \ldots, b_{t}^{m}\right)^{*}$ of mean rates of return is assumed to be $\mathcal{F}^{B}$-progressively measurable and $L$-Lipschitz uniformly in time, i.e.

$$
\left|b_{t}^{i}\left(\omega^{1}\right)-b_{t}^{i}\left(\omega^{2}\right)\right| \leq L \sum_{k=1}^{d} \sup _{0 \leq s \leq t}\left|\omega_{s}^{1, k}-\omega_{s}^{2, k}\right| \quad \forall t, i \text { and } \forall \omega^{1}, \omega^{2} \in \mathcal{C}^{d} .
$$

The $m \times d$ volatility matrix $\sigma_{t}=\left(\sigma_{t}^{i, j}\right)_{1 \leq i \leq m, 1 \leq j \leq d}$ has full rank, it is $\mathcal{F}^{B}$-progressively measurable and $M$-Lipschitz uniformly in time, i.e.

$$
\left|\sigma_{t}^{i, j}\left(\omega^{1}\right)-\sigma_{t}^{i, j}\left(\omega^{2}\right)\right| \leq M \sum_{k=1}^{d} \sup _{0 \leq s \leq t}\left|\omega_{s}^{1, k}-\omega_{s}^{2, k}\right| \quad \forall t, i, j \text { and } \forall \omega^{1}, \omega^{2} \in \mathcal{C}^{d},
$$

and there exists some constant $C$ s.t. $\left|\sigma_{t}^{i, j}\right| \leq C$ for each time $t$ and for any $i, j$. We denote by $\lambda_{t}^{i}$ the proportion of an agent's wealth invested in the $i$ th stock at time $t(1 \leq i \leq m)$, the remaining proportion $1-\sum_{i=1}^{m} \lambda_{t}^{i}$ being invested in the bond. We shall forbid short-selling of stocks and bond, which corresponds to the constraint $\lambda_{i} \in[0,1]$ for all $i$, and $\sum_{i=1}^{m} \lambda_{t}^{i} \leq 1$. We write $\lambda \in \mathbb{A}$ for this constraint; the case of arbitrary compact-convex constraints can be treated in the same way. Let $\mathcal{A}\left(\mathcal{G}^{B}\right)$ and $\mathcal{A}\left(\mathcal{F}^{B}\right)$ be the sets of admissible portfolios for the agent with and without anticipative information, i.e. the sets of $\mathcal{G}^{B}$-, respectively $\mathcal{F}^{B}$-progressively measurable $\mathbb{A}$-valued processes $\left(\lambda_{t}\right)_{t \in[0, T]}$. Denoting by $X^{\lambda}$ the wealth process corresponding to a portfolio $\lambda$ and starting from a unit of capital, we have $d X_{t}^{\lambda}=X_{t}^{\lambda} \lambda_{t}^{*}\left[b_{t} d t+\sigma_{t} d B_{t}\right]$, that is,

$$
X_{t}^{\lambda}=\exp \left(\int_{0}^{t}\left(\lambda_{s}^{*} b_{s}-\frac{1}{2}\left\|\sigma_{s}^{*} \lambda_{s}\right\|^{2}\right) d s+\int_{0}^{t} \lambda_{s}^{*} \sigma_{s} d B_{s}\right) .
$$


The above expression makes sense for portfolios in $\mathcal{A}\left(\mathcal{G}^{B}\right)$ by Assumption 4.6 and from the fact that $\mathbb{A}$ is bounded. We also need:

Assumption 4.7. The utility function $U: \mathbb{R}_{+} \rightarrow \mathbb{R}$ is concave, increasing, and such that, for some $K \in \mathbb{R}_{+}$, we have $F:=U \circ \exp$ is K-Lipschitz, concave and increasing.

We remark that this assumption is fulfilled e.g. by $U$ negative power utility $U(x)=\frac{x^{a}}{a}$ for $a \leq 0$, or logarithmic utility $U(x)=\ln (x)$, or exponential utility $U(x)=-\frac{1}{a} e^{-a x}$ for $a \geq 1$. The function $F$ is 1-Lipschitz for the first two examples, and $e^{-a}$-Lipschitz for the last one.

The utility maximization problem without anticipation of information is then given by

$$
v^{\mathcal{F}}=\sup _{\lambda \in \mathcal{A}\left(\mathcal{F}^{B}\right)} \mathbb{E}\left[U\left(X_{T}^{\lambda}\right)\right]
$$

We proceed to compare this value with the following problem under anticipation of information:

$$
v^{\mathcal{G}}=\sup _{\lambda \in \mathcal{A}\left(\mathcal{G}^{B}\right)} \mathbb{E}\left[U\left(X_{T}^{\lambda}\right)\right]
$$

hence obtaining a bound on the price of information relative to the risk-attitude encoded by $U$.

Proposition 4.8. The difference between the value functions of informed and uninformed agents can be bounded as follows (with the convention $+\infty-\infty=0$ )

$$
0 \leq v^{\mathcal{G}}-v^{\mathcal{F}} \leq \tilde{K} \inf _{\pi \in \Pi^{\mathcal{F}, \mathcal{G}}(\gamma, \gamma)} \mathbb{E}^{\pi}\left[V_{T}(\bar{\omega}-\omega)\right],
$$

for some explicit constant $\tilde{K}$, see (4.11).

We recall that by the total variation of an $\mathbb{R}^{d}$-valued process $X$ we mean the sum of total variations of its components, i.e. $V_{T}(X)=\sum_{i=1}^{d} V_{T}\left(X_{i}\right)$. Thanks to the multidimensional version of Theorem 3.2 (see Remark 3.3), we have that if the causal problem in (4.9) is finite, then its value equals $\mathbb{E}^{\gamma}\left[V_{T}(A)\right]$.

Proof. In the case $v^{\mathcal{F}}=\infty$ we do not have anything to prove, hence we assume $v^{\mathcal{F}}$ to be finite. In the path space $\mathcal{C}^{d}$, the expected utility from terminal wealth for the agents with and without anticipative information is given by

$$
\mathbb{E}^{\gamma}\left[F\left(\int_{0}^{T}\left(\lambda_{t}^{*} b_{t}-\frac{1}{2}\left\|\sigma_{t}^{*} \lambda_{t}\right\|^{2}\right) d t+\int_{0}^{T} \lambda_{t}^{*} \sigma_{t} d \omega_{t},\right)\right]
$$

where $\lambda$ is $\mathcal{G}$ - and $\mathcal{F}$-progressively measurable, respectively.

We now fix a causal transport $\pi \in \Pi^{\mathcal{F}, \mathcal{G}}(\gamma, \gamma) \subset \mathcal{P}\left(C^{d} \times \mathcal{C}^{d}\right)$ for which the total variation $V_{T}(\bar{\omega}-\omega)$ is $\pi$-a.s. finite, and consider $(U(\mathcal{F}))$ to be solved in the $\omega$ variable and $(U(\mathcal{G}))$ in the $\bar{\omega}$ variable. Assume $v^{\mathcal{G}}<\infty$ and that there is an optimizer $\hat{\lambda}(\bar{\omega})=\left(\hat{\lambda}^{1}(\bar{\omega}), \ldots, \hat{\lambda}^{m}(\bar{\omega})\right)$ for problem $\left(U(\mathcal{G})\right.$ ) (else one can argue in the same way for every element $\lambda^{n}$ of a sequence such that $\mathbb{E}\left[U\left(X_{T}^{\lambda^{n}}\right)\right] \rightarrow v^{\mathcal{G}}$ for $\left.n \rightarrow \infty\right)$. We denote by $\widetilde{\lambda}=\left(\widetilde{\lambda}^{1}, \ldots, \widetilde{\lambda}^{m}\right)$ its optional projection with respect to $(\pi, \mathcal{F} \otimes\{\emptyset, \mathcal{C}\})$, so that in particular

$$
\widetilde{\lambda}_{t}^{i}(\omega)=\widetilde{\lambda}_{t}^{i}(\omega, \bar{\omega})=\mathbb{E}^{\pi}\left[\hat{\lambda}_{t}^{i}(\bar{\omega}) \mid \mathcal{F}_{t}\right]=\mathbb{E}^{\pi}\left[\hat{\lambda}_{t}^{i}(\bar{\omega}) \mid \mathcal{F}_{T}\right], i=1 \ldots, m,
$$

(for simplicity, here and in what follows, we use the notation $\mathbb{E}^{\pi}\left[. \mid \mathcal{F}_{t}\right]$ for $\mathbb{E}^{\pi}\left[. \mid \mathcal{F}_{t} \otimes\{\emptyset, \mathcal{C}\}\right]$ ). The last equality follows by causality and is crucial for the next argument. Note that $\widetilde{\lambda} \in \mathcal{A}\left(\mathcal{F}^{B}\right)$, 
which yields

$$
\begin{aligned}
v^{\mathcal{F}} & \geq \mathbb{E}^{\gamma}\left[F\left(\int_{0}^{T}\left(\widetilde{\lambda}_{t}^{*}(\omega) b_{t}(\omega)-\frac{1}{2}\left\|\sigma_{t}^{*}(\omega) \widetilde{\lambda}_{t}(\omega)\right\|^{2}\right) d t+\int_{0}^{T} \widetilde{\lambda}_{t}^{*}(\omega) \sigma_{t}(\omega) d \omega_{t}\right)\right] \\
& \geq \mathbb{E}^{\pi}\left[F\left(\mathbb{E}^{\pi}\left[\int_{0}^{T}\left(\hat{\lambda}_{t}^{*}(\bar{\omega}) b_{t}(\omega)-\frac{1}{2}\left\|\sigma_{t}^{*}(\omega) \hat{\lambda}_{t}(\bar{\omega})\right\|^{2}\right) d t+\int_{0}^{T} \hat{\lambda}_{t}^{*}(\bar{\omega}) \sigma_{t}(\omega) d \omega_{t} \mid \mathcal{F}_{T}\right]\right)\right] \\
& \geq \mathbb{E}^{\pi}\left[F\left(\int_{0}^{T}\left(\hat{\lambda}_{t}^{*}(\bar{\omega}) b_{t}(\omega)-\frac{1}{2}\left\|\sigma_{t}^{*}(\omega) \hat{\lambda}_{t}(\bar{\omega})\right\|^{2}\right) d t+\int_{0}^{T} \hat{\lambda}_{t}^{*}(\bar{\omega}) \sigma_{t}(\omega) d \omega_{t}\right)\right]
\end{aligned}
$$

by Jensen's inequality, being $F$ concave and increasing, and by causality. Therefore, by Lipschitz continuity of the function $F$, we have

$$
\begin{array}{r}
\begin{array}{r}
0 v^{\mathcal{G}}-v^{\mathcal{F}} \leq K \mathbb{E}^{\pi}\left[\mid \int_{0}^{T} \hat{\lambda}_{t}^{*}(\bar{\omega})\left(b_{t}(\bar{\omega})-b_{t}(\omega)\right) d t-\frac{1}{2} \int_{0}^{T}\left(\left\|\left.\sigma_{t}^{*}(\bar{\omega}) \hat{\lambda}_{t}(\bar{\omega})\right|^{2}-\right\| \sigma_{t}^{*}(\omega) \hat{\lambda}_{t}(\bar{\omega}) \|^{2}\right) d t\right. \\
\left.+\int_{0}^{T} \hat{\lambda}_{t}^{*}(\bar{\omega})\left(\sigma_{t}(\bar{\omega}) d \bar{\omega}_{t}-\sigma_{t}(\omega) d \omega_{t}\right) \mid\right] \\
\leq K \mathbb{E}^{\pi}\left[\int_{0}^{T}\left|\hat{\lambda}_{t}^{*}(\bar{\omega})\left(b_{t}(\bar{\omega})-b_{t}(\omega)\right)\right| d t+\left|\int_{0}^{T} \hat{\lambda}_{t}^{*}(\bar{\omega})\left(\sigma_{t}(\bar{\omega}) d \bar{\omega}_{t}-\sigma_{t}(\omega) d \omega_{t}\right)\right|\right. \\
\left.+\frac{1}{2} \int_{0}^{T} \sum_{j=1}^{d}\left[\sum_{i=1}^{m}\left|\hat{\lambda}_{t}^{i}(\bar{\omega})\right|\left|\sigma_{t}^{i, j}(\bar{\omega})-\sigma_{t}^{i, j}(\omega)\right|\right]\left[\sum_{i=1}^{m}\left|\hat{\lambda}_{t}^{i}(\bar{\omega})\right|\left|\sigma_{t}^{i, j}(\bar{\omega})+\sigma_{t}^{i, j}(\omega)\right|\right] d t\right] \\
\leq K \mathbb{E}^{\pi}\left[\int_{0}^{T} \sum_{i=1}^{m}\left|b_{t}^{i}(\bar{\omega})-b_{t}^{i}(\omega)\right| d t+\left|\int_{0}^{T} \hat{\lambda}_{t}^{*}(\bar{\omega}) \sigma_{t}(\bar{\omega})\left(d \bar{\omega}_{t}-d \omega_{t}\right)\right|+\left|\int_{0}^{T} \hat{\lambda}_{t}^{*}(\bar{\omega})\left(\sigma_{t}(\bar{\omega})-\sigma_{t}(\omega)\right) d \omega_{t}\right|\right. \\
\left.\quad+\frac{1}{2} \int_{0}^{T} \sum_{j=1}^{d}\left[\sum_{i=1}^{m}\left|\sigma_{t}^{i, j}(\bar{\omega})-\sigma_{t}^{i, j}(\omega)\right|\right]\left[\sum_{i=1}^{m}\left|\sigma_{t}^{i, j}(\bar{\omega})+\sigma_{t}^{i, j}(\omega)\right|\right] d t\right] . \quad(4.10)
\end{array}
\end{array}
$$

Now, by (4.7) and since $\bar{\omega}_{0}-\omega_{0}=0$ on $\mathcal{C}^{d}$, we have that $\pi$-a.s.

$\int_{0}^{T} \sum_{i=1}^{m}\left|b_{t}^{i}(\bar{\omega})-b_{t}^{i}(\omega)\right| d t \leq \operatorname{LTm} \sum_{k=1}^{d} \sup _{0 \leq t \leq T}\left|\bar{\omega}_{t}^{k}-\omega_{t}^{k}\right| \leq \operatorname{LTm} \sum_{k=1}^{d} V_{T}\left(\bar{\omega}^{k}-\omega^{k}\right)=\operatorname{LTm} V_{T}(\bar{\omega}-\omega)$.

The second term in (4.10) is easily bounded $\pi$-a.s. as follows

$$
\left|\int_{0}^{T} \hat{\lambda}_{t}^{*}(\bar{\omega}) \sigma_{t}(\bar{\omega})\left(d \bar{\omega}_{t}-d \omega_{t}\right)\right|=\sum_{j=1}^{d} \sum_{i=1}^{m}\left|\int_{0}^{T} \hat{\lambda}_{t}^{i}(\bar{\omega}) \sigma_{t}^{i, j}(\bar{\omega})\left(d \bar{\omega}_{t}^{j}-d \omega_{t}^{j}\right)\right| \leq C m V_{T}(\bar{\omega}-\omega) .
$$

Finally we consider the third term in (4.10), and denote $X_{t}=\int_{0}^{t} \hat{\lambda}_{s}^{*}(\bar{\omega})\left(\sigma_{s}(\bar{\omega})-\sigma_{s}(\omega)\right) d \omega_{s}$. This is a martingale under $\pi$, because by causality the coordinate process $\omega$ is an $\mathcal{F} \otimes \mathcal{G}$-martingale. Hence, we can apply the Burkholder-Davis-Gundy inequality, obtaining

$$
\begin{aligned}
\mathbb{E}^{\pi}\left[\left|X_{T}\right|\right] & \leq C_{1} \mathbb{E}^{\pi}\left[\sqrt{\langle X, X\rangle_{T}}\right] \\
& =C_{1} \mathbb{E}^{\pi}\left[\sqrt{\int_{0}^{T} \sum_{j=1}^{d}\left|\sum_{i=1}^{m} \hat{\lambda}_{t}^{i}(\bar{\omega})\left(\sigma_{t}^{i, j}(\bar{\omega})-\sigma_{t}^{i, j}(\omega)\right)\right|^{2} d t}\right] \\
& \leq C_{1} \sqrt{m} \mathbb{E}^{\pi}\left[\sqrt{\left.\int_{0}^{T} \sum_{j=1}^{d} \sum_{i=1}^{m}\left|\sigma_{t}^{i, j}(\bar{\omega})-\sigma_{t}^{i, j}(\omega)\right|^{2} d t\right]}\right. \\
& \leq C_{1} M \sqrt{m d} \mathbb{E}^{\pi}\left[\sqrt{\int_{0}^{T}\left\{\sum_{k=1}^{d} \sup _{0 \leq s \leq t}\left|\bar{\omega}_{s}^{k}-\omega_{s}^{k}\right|\right\}^{2} d t}\right] \\
& \leq C_{1} M \sqrt{T m d} \mathbb{E}^{\pi}\left[\sum_{k=1}^{d} \sup _{0 \leq s \leq T}\left|\bar{\omega}_{s}^{k}-\omega_{s}^{k}\right|\right] \\
& \leq C_{1} M \sqrt{T m d} \mathbb{E}^{\pi}\left[V_{T}(\bar{\omega}-\omega)\right] .
\end{aligned}
$$


Hence, the difference $v^{\mathcal{G}}-v^{\mathcal{F}}$ is bounded above by $\tilde{K} \mathbb{E}^{\pi}\left[V_{T}(\bar{\omega}-\omega)\right]$, with

$$
\tilde{K}=K\left(L T m+m^{2} d C M T+C m+C_{1} M \sqrt{T d m}\right) .
$$

Since $\pi$ was a generic transport in $\Pi^{\mathcal{F}, \mathcal{G}}(\gamma, \gamma)$, this concludes the proof for the case $v^{\mathcal{G}}<\infty$. The case $v^{\mathcal{G}}=\infty$ follows similarly, working along a sequence $\lambda^{n}$ s.t. $\mathbb{E}\left[U\left(X_{T}^{\lambda^{n}}\right)\right] \rightarrow \infty$.

Remark 4.9. In the proof of Proposition 4.8, the only place where we use the fact that one filtration is bigger than the other is to state that the difference of the two utility maximization problems is non-negative. Thus all estimates in (4.9), except the leftmost one, can be obtained in the same way for agents with any sets of informations (non-necessarily one bigger than the other).

\section{$5 \quad$ Attainability and Duality}

\subsection{Classical and constrained transport: an extension}

In this section we consider the general abstract setting of Section 2.1. As we have seen in previous sections, it is important to obtain attainability and duality results for (2.1), and more specifically (2.2), when the cost function $c$ is Borel measurable with values in the extended real line $(-\infty,+\infty]$; e.g. for Cameron-Martin or total-variation costs. For such problems there is no systematic theory, and indeed [BS11, Example 4.1] shows that duality may fail in such a setting. Fortunately, the cost functions of interest in this article have a strong structural property. Assuming this property will allow us to prove attainability/duality results for (2.1)(2.2) in a simple and self-contained way. The next result can also be proven via the following argument: on every Polish space, there is a finer Polish topology having the same Borel sets, for which a given real-valued Borel function becomes continuous. This argument, however, would not lead us to prove Corollary 5.3 below, nor help us studying our ultimate object, namely (2.2). So we rather give our own arguments below.

Proposition 5.1. Let $f: \mathcal{X} \rightarrow \mathbb{R}$ and $g: \mathcal{Y} \rightarrow \mathbb{R}$ be bounded Borel functions and $\tilde{c}: \mathcal{X} \times \mathcal{Y} \rightarrow$ $(-\infty, \infty]$ be lower semicontinuous and bounded from below. Suppose that either $f$ or $g$ is further continuous, and define

$$
c(x, y):=\tilde{c}(x, y)+f(x) g(y) .
$$

Then the optimal transport problem (2.1) corresponding to the cost c is attained. Furthermore, there is no duality gap:

$$
\inf _{\pi \in \Pi(\mu, \nu)} \mathbb{E}^{\pi}[c]=\sup _{\substack{\phi \in \mathcal{C}_{b}(\mathcal{X}), \psi \in \mathcal{C}_{b}(\mathcal{Y}) \\ \phi \oplus \psi \leq \tilde{c}+f g}}\left\{\mathbb{E}^{\mu}[\phi]+\mathbb{E}^{\nu}[\psi]\right\} .
$$

Before providing the proof of the above proposition, we introduce the following lemma.

Lemma 5.2. Assume that $\mathcal{X}, \mathcal{Y}$ are Polish spaces equipped with Borel probability measures $\mu, \nu$. Let $f: \mathcal{X} \rightarrow \mathbb{R}$ and $g: \mathcal{Y} \rightarrow \mathbb{R}$ be bounded Borel function, at least one of which is continuous. Then the function

$$
\pi \mapsto \mathbb{E}^{\pi}[f(x) g(y)]
$$

is continuous on $\Pi(\mu, \nu)$ with respect to the weak topology. 
Proof. Recall that $\Pi(\mu, \nu)$ is a compact subset of $\mathcal{P}(\mathcal{X} \times \mathcal{Y})$ with respect to the weak topology. W.l.o.g. we assume that $f$ is continuous and let $M \in \mathbb{R}$ be such that $|f| \leq M$, and $g_{k}: \mathcal{Y} \rightarrow$ $\mathbb{R}, k \in \mathbb{N}$, be a sequence of bounded continuous functions converging to $g$ in $L^{1}(\nu)$. Consider a sequence of measures $\left(\pi_{n}\right)_{n} \subseteq \Pi(\mu, \nu)$ such that $\pi_{n}$ converges weakly to $\pi$ for some $\pi \in \Pi(\mu, \nu)$. For any $\epsilon>0$, take $k(\epsilon)$ such that $\left\|g-g_{k}\right\|_{L^{1}(\nu)} \leq \epsilon /(2 M+1)$ for all $k \geq k(\epsilon)$, and take $n(\epsilon)$ such that $\left|\mathbb{E}^{\pi}\left[f g_{k(\epsilon)}\right]-\mathbb{E}^{\pi_{n}}\left[f g_{k(\epsilon)}\right]\right| \leq \epsilon /(2 M+1)$ for all $n \geq n(\epsilon)$. Then, for all $n \geq n(\epsilon)$,

$$
\begin{aligned}
\left|\mathbb{E}^{\pi}[f g]-\mathbb{E}^{\pi_{n}}[f g]\right| & \leq \mathbb{E}^{\pi}\left[|f|\left|g-g_{k(\epsilon)}\right|\right]+\left|\mathbb{E}^{\pi}\left[f g_{k(\epsilon)}\right]-\mathbb{E}^{\pi_{n}}\left[f g_{k(\epsilon)}\right]\right|+\mathbb{E}^{\pi_{n}}\left[|f|\left|g-g_{k(\epsilon)}\right|\right] \\
& \leq M \epsilon /(2 M+1)+\epsilon /(2 M+1)+M \epsilon /(2 M+1) \leq \epsilon,
\end{aligned}
$$

which proves the desired statement.

Proof of Proposition 5.1. We employ classical arguments, as in [Vil03] or [BS11, Sect. 1.3]. Since $\tilde{c}$ is lower semicontinuous, there exists a sequence $\left(\tilde{c}_{n}\right)_{n}$ of bounded continuous functions on $\mathcal{X} \times \mathcal{Y}$ such that $\tilde{c}_{n} \uparrow \tilde{c}$. We are going to show that $P\left(c_{n}\right):=\inf _{\pi \in \Pi(\mu, \nu)} \mathbb{E}^{\pi}\left[c_{n}\right]$ converges to $P(c):=\inf _{\pi \in \Pi(\mu, \nu)} \mathbb{E}^{\pi}[c]$, where $c_{n}(x, y)=\tilde{c}_{n}(x, y)+f(x) g(y)$. In order to do so, for each $n \in \mathbb{N}$, we pick $\pi_{n} \in \Pi(\mu, \nu)$ such that $\mathbb{E}^{\pi_{n}}\left(c_{n}\right) \leq P\left(c_{n}\right)+1 / n$. Since $\Pi(\mu, \nu)$ is weakly compact, we may assume that $\left(\pi_{n}\right)_{n}$ converges weakly to some transport plan $\pi \in \Pi(\mu, \nu)$. Then,

$$
\begin{aligned}
P(c) & \leq \mathbb{E}^{\pi}[c]=\lim _{m} \mathbb{E}^{\pi}\left[c_{m}\right]=\lim _{m}\left(\lim _{n} \mathbb{E}^{\pi_{n}}\left[c_{m}\right]\right) \leq \lim _{m}\left(\lim _{n} \mathbb{E}^{\pi_{n}}\left[c_{n}\right]\right) \\
& =\lim _{n} \mathbb{E}^{\pi_{n}}\left[c_{n}\right]=\lim _{n} P\left(c_{n}\right) \leq P(c),
\end{aligned}
$$

where we used monotone convergence, Lemma 5.2 to ensure that $\mathbb{E}^{\pi}\left[c_{m}\right]=\lim _{n} \mathbb{E}^{\pi_{n}}\left[c_{m}\right]$, and the facts that $c_{n}$ is an increasing sequence with $P\left(c_{n}\right) \leq \mathbb{E}^{\pi_{n}}\left(c_{n}\right) \leq P\left(c_{n}\right)+1 / n$. This concludes the proof of our claim and actually shows that $\pi$ is an optimizer for the cost $c$ (this easily follows by compactness and Lemma 5.2). The function $c_{n}$ is Borel bounded, so by [Kel84, Theorem 2.14] we have that duality holds for it. Thus we can pick $\left(\psi_{n}, \phi_{n}\right)$ such that $\mathbb{E}^{\mu}\left[\psi_{n}\right]+\mathbb{E}^{\nu}\left[\phi_{n}\right] \geq P\left(c_{n}\right)-1 / n$, hence $\sup _{n}\left(\mathbb{E}^{\mu}\left[\psi_{n}\right]+\mathbb{E}^{\nu}\left[\phi_{n}\right]\right) \geq \lim _{n} P\left(c_{n}\right)-1 / n=P(c)$. Since $\psi_{n}(x)+\phi_{n}(y) \leq c_{n}(x, y) \leq c(x, y)$, duality is established.

It is clear that in Proposition 5.1 one can take $c$ to contain a finite sum of terms of the form $f(x) g(y)$ as described. We give now a corollary of this proposition, dealing with a class of optimal transport problems under linear constraints. It is this result that we shall apply to the setting of causal optimal transport. We refer to [Zae15, BG19] for more on linearly constrained transport problems, but remark that the result below is not a consequence of theirs.

Corollary 5.3. Let $\mathfrak{F}$ (resp. $\mathfrak{G}$ ) be a non-empty collection of real-valued bounded Borel functions on $\mathcal{X}$ (resp. $\mathcal{Y})$, and define $\mathfrak{H}^{\mathfrak{F}, \mathfrak{G}}:=\operatorname{span}\{f g: f \in \mathfrak{F}, g \in \mathfrak{G}\}$. We define the optimal transport with linear constraints determined by $\mathfrak{H}^{\mathfrak{F}, \mathfrak{G}}$ as

$$
\inf _{\substack{\pi \in \Pi(\mu, \nu) \\ \forall h \in \mathfrak{H}^{\mathfrak{r}, \mathfrak{S}}: \mathbb{E}^{\pi}[h]=0}} \mathbb{E}^{\pi}[c]
$$

Assume that $c: \mathcal{X} \times \mathcal{Y} \rightarrow(-\infty, \infty]$ is lower semicontinuous and bounded from below, and that either all elements of $\mathfrak{F}$, or all elements of $\mathfrak{G}$, are continuous. Then (5.1) is attained, and there is no duality gap:

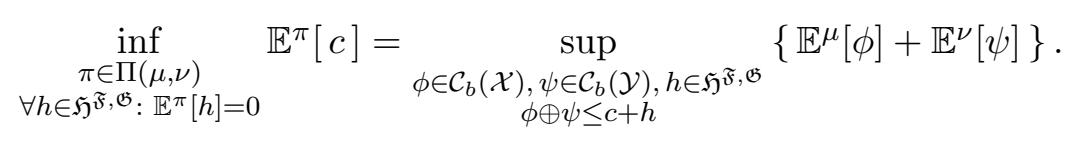


Proof. The set of all $\pi \in \Pi(\mu, \nu)$ s.t. for all $h \in \mathfrak{H}^{\mathfrak{F}, \mathfrak{G}}$ it holds that $\mathbb{E}^{\pi}[h]=0$, is a weakly closed subset of the compact set $\Pi(\mu, \nu)$ (this follows from Lemma 5.2). This is enough to ensure the attainability of (5.1). Further, it is immediate that

$$
\inf _{\substack{\pi \in \Pi(\mu, \nu) \\ \forall h \in \mathfrak{H}^{\mathfrak{F}, \mathfrak{G}}: \mathbb{E}^{\pi}[h]=0}} \mathbb{E}^{\pi}[c]=\inf _{\pi \in \Pi(\mu, \nu)} \sup _{h \in \mathfrak{H}^{\mathfrak{r}, \mathfrak{G}}} \mathbb{E}^{\pi}[c+h]=\sup _{h \in \mathfrak{H}^{\mathfrak{F}, \mathfrak{G}}} \inf _{\pi \in \Pi(\mu, \nu)} \mathbb{E}^{\pi}[c+h],
$$

by the usual minimax arguments (e.g. [Sio58], observing that the affine bilinear objective functions is lower semicontinuous in $\pi$ by Lemma 5.2, as $\pi$ varies over a compact). By Proposition 5.1,

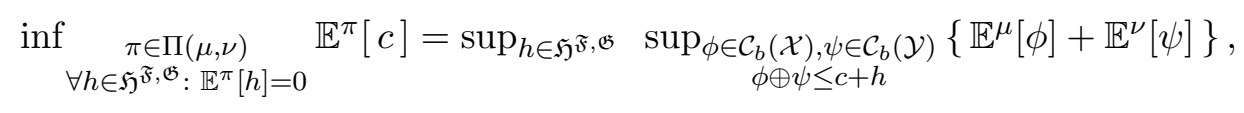

yielding the desired result.

\subsection{Attainability and Duality in causal transport}

We recall from (2.2) that by a causal optimal transport problem with respect to a cost function $c: \mathcal{X} \times \mathcal{Y} \rightarrow(-\infty, \infty]$, we mean the optimization problem

$$
\inf _{\pi \in \Pi^{\mathcal{X}}, \mathcal{F}^{\mathcal{Y}}} \mathbb{E}_{(\mu, \nu)}^{\pi}[c]
$$

It has already been observed that these problems form a subclass of optimal transport problems under linear constraints; see [Las15, BBLZ17]. Let us make this precise in the present setting, by defining

$$
\mathfrak{H}:=\operatorname{span}\left(\left\{g\left[f-\mathbb{E}^{\mu}\left[f \mid \mathcal{F}_{t}^{\mathcal{X}}\right]\right]: f \in C_{b}(\mathcal{X}), g \in B_{b}\left(\mathcal{Y}, \mathcal{F}_{t}^{\mathcal{Y}}\right), t \in[0, T]\right\}\right) .
$$

Lemma 5.4. Let $\pi \in \Pi(\mu, \nu)$. Then $\pi$ is causal between $\mathcal{F}^{\mathcal{X}}$ and $\mathcal{F}^{\mathcal{Y}}\left(\right.$ i.e. $\left.\pi \in \Pi^{\mathcal{F}^{\mathcal{X}}, \mathcal{F}^{\mathcal{Y}}}(\mu, \nu)\right)$ if and only if $\mathbb{E}^{\pi}[h]=0$ for all $h \in \mathfrak{H}$.

Proof. For $g$ bounded $\left(\mathcal{Y}, \mathcal{F}_{t}^{\mathcal{Y}}\right)$-measurable, denote $g^{t}(x):=\mathbb{E}^{\pi}\left[g(y) \mid \mathcal{F}_{T}^{\mathcal{X}} \otimes\{\emptyset, \mathcal{C}\}\right](x)$. By definition, $\pi$ is causal between $\mathcal{F}^{\mathcal{X}}$ and $\mathcal{F}^{\mathcal{Y}}$ if and only if for all $t \leq T$ and all such $g^{t}$ we have

$$
g^{t}=\mathbb{E}^{\mu}\left[g^{t} \mid \mathcal{F}_{t}^{\mathcal{X}}\right], \quad \mu-a . s,
$$

which is equivalent to

$$
\mathbb{E}^{\mu}\left[f\left(g^{t}-\mathbb{E}^{\mu}\left[g^{t} \mid \mathcal{F}_{t}^{\mathcal{X}}\right]\right)\right]=0,
$$

for every continuous bounded function $f: \mathcal{X} \rightarrow \mathbb{R}$ and for all $t \leq T$. The fact that we can take the $f$ 's continuous and not merely measurable comes from the fact that $\mu$ is a Borel finite measure on a Polish space. It is easy to see that the previous equation is equivalent to

$$
\mathbb{E}^{\mu}\left[g^{t}\left(f-\mathbb{E}^{\mu}\left[f \mid \mathcal{F}_{t}^{\mathcal{X}}\right]\right)\right]=0 .
$$

Finally, by the tower property of conditional expectations, the latter is in turn equivalent to

$$
\mathbb{E}^{\pi}\left[g\left(f-\mathbb{E}^{\mu}\left[f \mid \mathcal{F}_{t}^{\mathcal{X}}\right]\right)\right]=0 .
$$


In [Las15, Sect. 3] the author proves, in the general setting of Polish spaces, that the set $\Pi^{\mathcal{F}^{\mathcal{X}}, \mathcal{F}^{\mathcal{Y}}}(\mu, \nu)$ is closed for weak convergence. Thus an attainability and duality theory for the problem of optimal transport under the causality constraint follows. This is done there at the expense of a regularity assumptions of sorts on the filtration $\mathcal{F}^{\mathcal{Y}}$ (see [Las15, Definition 3]). Such an assumption would, in our context, drastically limit the applicability of the transport approach. As we now show, we can still obtain an attainability and duality theory without assumptions on $\mathcal{F}^{\mathcal{Y}}$. We do this at the price of requiring the first marginal $\mu$ to be "weakly continuous" in a precise sense. This is enough for the purpose of our work.

Lemma 5.5. Assume that $\mu$ satisfies the following weak continuity property:

$$
\forall f \in C_{b}(\mathcal{X}), t \in[0, T]: x \mapsto \mathbb{E}^{\mu}\left[f \mid \mathcal{F}_{t}^{\mathcal{X}}\right](x) \text { is continuous. }
$$

Then the set $\Pi^{\mathcal{F}^{\mathcal{X}}, \mathcal{F}^{\mathcal{Y}}}(\mu, \nu)$ of causal couplings is compact for weak convergence.

Proof. Since $\Pi^{\mathcal{F}^{\mathcal{X}}, \mathcal{F}^{\mathcal{Y}}}(\mu, \nu) \subseteq \Pi(\mu, \nu)$, and the latter is weakly compact, we only need to show that $\Pi^{\mathcal{F}^{\mathcal{X}}, \mathcal{F}^{\mathcal{Y}}}(\mu, \nu)$ is weakly closed. This follows from Lemma 5.4, since the condition $\mathbb{E}^{\pi}[h]=0$ is closed for all $h \in \mathfrak{H}$, by Lemma 5.2 and the continuity property (5.2).

Theorem 5.6 (Causal transport duality). Let $c: \mathcal{X} \times \mathcal{Y} \rightarrow(-\infty, \infty]$ be bounded from below and lower semicontinuous. Further assume that $\mu$ satisfies the weak continuity property (5.2). Then (2.2) is attained and there is no duality gap:

$$
\inf _{\pi \in \Pi^{\mathcal{F}}, \mathcal{F}^{\mathcal{Y}}(\mu, \nu)} \mathbb{E}^{\pi}[c]=\sup _{\substack{\phi \in C_{b}(\mathcal{X}), \psi \in C_{b}(\mathcal{Y}), h \in \mathfrak{H} \\ \phi \oplus \psi \leq c+h}}\left\{\mathbb{E}^{\mu}[\phi]+\mathbb{E}^{\nu}[\psi]\right\}=\sup _{\substack{\psi \in C_{b}(\mathcal{Y}), h \in \mathfrak{H} \\ \psi \leq c+h}} \mathbb{E}^{\nu}[\psi] .
$$

Proof. Attainability follows by classical arguments from Lemma 5.5. Duality is a direct consequence of Lemma 5.4 and Corollary 5.3, upon observing that $\mathfrak{H}$ has the correct structure and that, under the weak continuity assumption on $\mu$, all the $x$-dependent factors generating $\mathfrak{H}$ are continuous and bounded. The fact that $\phi$ disappears from the dual problem follows from the fact that $\phi-\mathbb{E}^{\mu}[\phi]$ belongs to $\mathfrak{H}$.

The previous weak continuity property of $\mu$ is fulfilled if e.g. $\mathcal{X}$ is a path space and $\mu$ is the law of a Feller process. So the case that interests us, Wiener measure on the space of continuous paths, is fully covered.

\section{A Elements of Orlicz space theory}

As presented in [RR91], a convex even function $\Phi: \mathbb{R} \rightarrow \mathbb{R}_{+} \cup\{+\infty\}$ satisfying $\Phi(0)=0$ and $\Phi(\infty)=\infty$, is called a Young function. If such a function is finite-valued, it is zero only at the origin, and satisfies both $\Phi(0) / 0=0$ and $\Phi(\infty) / \infty=\infty$, then it is called an $N$-function. We remark that $\Phi$ is a Young function (resp. N-function) if and only if its conjugate $\Phi^{*}$ is so.

From now on we identify processes which are $d \nu \times d t$-a.e. equal. Assuming that $\Phi$ is a Young function, we define

$$
M^{\Phi}:=\left\{F: \mathcal{C} \times[0, T] \rightarrow \mathbb{R} \text {, s.t. } F \text { is } \mathcal{G} \text {-previsible and } \forall k>0: \mathbb{E}^{\nu}\left[\int_{0}^{T} \Phi\left(k F_{t}\right) d t\right]<\infty\right\},
$$

which is a closed subspace (sometimes called Orlicz heart or Morse-Transue space) of the socalled Orlicz space

$$
L^{\Phi}:=\left\{F: \mathcal{C} \times[0, T] \rightarrow \mathbb{R}, \text { s.t. } F \text { is } \mathcal{G} \text {-previsible and } \exists k>0: \mathbb{E}^{\nu}\left[\int_{0}^{T} \Phi\left(k F_{t}\right) d t\right]<\infty\right\},
$$


when endowed with the gauge norm

$$
\|F\|_{\Phi}:=\inf \left\{\beta>0: \mathbb{E}^{\nu}\left[\int_{0}^{T} \Phi\left(F_{t} / \beta\right) d t\right] \leq 1\right\} .
$$

The gauge norm actually turns $L^{\Phi}$ into a Banach space, and if e.g. $\Phi$ is an N-function then the norm-dual of $M^{\Phi}$ is $L^{\Phi^{*}}$ ([RR91, Ch. III.3.3, Theorem 10] and [RR91, Ch. IV.4.1, Theorem 6]).

We now introduce growth conditions on $\Phi$ and $\Phi^{*}$. We say that a Young function $\Phi$ is in $\Delta_{2}$ if there are some constants $C, x_{0}>0$ s.t. whenever $x \geq x_{0}$ we have $\Phi(2 x) \leq C \Phi(x)$. This is seen equivalent to the following condition on $\Phi^{*}$ : there exist $\ell>1, x_{0}>0$ s.t. whenever $x \geq x_{0}$ we have $\Phi^{*}(x) \leq \frac{\Phi^{*}(\ell x)}{2 \ell}$. When $\Phi$ is an N-function, then by [RR91, Ch. II.2.3, Theorem 3]:

$$
\Phi \text { in } \Delta_{2} \Longleftrightarrow \exists x_{0}>0, \epsilon>1 \text { s.t. }\left[x \geq x_{0} \Rightarrow \frac{x \Phi^{\prime}(x)}{\Phi(x)} \leq \epsilon\right] .
$$

Clearly when $\Phi$ is in $\Delta_{2}$ we have $M^{\Phi}=L^{\Phi}$. The reflexivity of $L^{\Phi}$ is essentially equivalent to $\Phi$ and $\Phi^{*}$ being in $\Delta_{2}$.

We finally provide a technical lemma useful in the proof of Theorem 3.8:

Lemma A.1. If $\rho^{*}$ is an $N$-function in $\Delta_{2}$, then

$$
(\omega, \bar{\omega}) \in \mathcal{C} \times \mathcal{C} \mapsto \int_{0}^{T} \rho\left(\left(\frac{\dot{\bar{\omega}-\omega}}{t}\right) t t\right.
$$

is lower semicontinuous when $\mathcal{C} \times \mathcal{C}$ is equipped with the supremum norm.

Proof. It suffices to show the lower semicontinuity of $\bar{\omega} \mapsto r(\bar{\omega}):=\int_{0}^{T} \rho\left(\dot{\bar{\omega}}_{t}\right) d t$, where $\dot{\bar{\omega}}_{t}$ is the density of $\bar{\omega}$, if $\bar{\omega}$ is absolutely continuous, and equals $+\infty$ otherwise. Let $\mathcal{C}_{K}:=\{\bar{\omega} \in \mathcal{C}: r(\bar{\omega}) \leq$ $K\}$. Since $\rho^{*}$ is in $\Delta_{2}$, we have by [RR91, Ch. II.2.3, Corollary 5] that $\rho$ grows at least as fast as some power function with exponent $p>1$. This shows that $\mathcal{C}_{K}$ is bounded in $W^{1, p}([0, T])$, the Sobolev space of absolutely continuous functions with $p$-integrable first derivative. By classical arguments we have that if $\bar{\omega}^{n} \rightarrow \bar{\omega}$ uniformly, with $\bar{\omega}^{n} \in \mathcal{C}_{K}$, then $\bar{\omega} \in W^{1, p}([0, T])$ and in particular $\bar{\omega}$ is absolutely continuous too. By Fatou's Lemma we further get $\bar{\omega} \in \mathcal{C}_{K}$, yielding the desired result.

\section{B Proof of Theorem 3.8}

We consider a fixed measure $\nu$ on $\mathcal{C}$. The idea behind the refined dual (3.10) comes from the next argument:

Lemma B.1. Let $\rho: \mathbb{R} \rightarrow \mathbb{R} \cup\{+\infty\}$ be convex. Weak-refined duality holds, in the sense that the value of the primal problem (3.6) can only be larger than that of the refined dual (3.10).

Proof. W.l.o.g. we assume that (3.6) is finite. Let $F \in S_{a}(\mathcal{G})$. For any pair $(\omega, \bar{\omega})$ whose difference is absolutely continuous, with derivative $\alpha_{t}$, the Fenchel-Young inequality gives

$$
\rho\left(\alpha_{t}\right) \geq F_{t}(\bar{\omega}) \alpha_{t}-\rho^{*}\left(F_{t}(\bar{\omega})\right),
$$

so that integrating in time yields

$$
\begin{aligned}
\int_{0}^{T} \rho\left(\alpha_{t}\right) d t & \geq \int_{0}^{T} F_{t}(\bar{\omega}) \alpha_{t} d t-\int_{0}^{T} \rho^{*}\left(F_{t}(\bar{\omega})\right) d t \\
& =\int_{0}^{T} F_{t}(\bar{\omega}) d(\bar{\omega}-\omega)_{t}-\int_{0}^{T} \rho^{*}\left(F_{t}(\bar{\omega})\right) d t .
\end{aligned}
$$


Now, taking expectation under any $\pi \in \Pi^{\mathcal{F}, \mathcal{G}}(\gamma, \nu)$ with finite cost for the problem in (3.6), we obtain by causality

$$
\mathbb{E}^{\pi}\left[\int_{0}^{T} \rho\left((\widehat{\overline{\omega-\omega}})_{t}\right) d t\right] \geq \mathbb{E}^{\nu}\left[\int_{0}^{T} F_{t}(\bar{\omega}) d \bar{\omega}-\int_{0}^{T} \rho^{*}\left(F_{t}(\bar{\omega})\right)\right] .
$$

We conclude that the value of the primal problem is not any less than (3.10).

We now define a stochastic integral; see Appendix A for terminology and notation on Orlicz spaces, such as "N- and Young-functions", the " $\Delta_{2}$ condition" and so forth.

Lemma B.2. Suppose that $\rho^{*}$ is a Young function having a global minimum at the origin. Then

$$
\overline{S_{a}(\mathcal{G})} \|^{\| \cdot \rho^{*}}=M^{\rho^{*}} .
$$

If further the refined dual (3.10) is finite, then the functional

$$
F=\sum F^{i} \mathbb{I}_{\left(\tau_{i}, \tau_{i+1}\right]} \in S_{a}(\mathcal{G}) \mapsto \mathbb{E}^{\nu}\left[\int_{0}^{T} F_{t}(\bar{\omega}) d \bar{\omega}_{t}\right]:=\mathbb{E}^{\nu}\left[\sum F^{i}(\bar{\omega})\left(\bar{\omega}_{\tau_{i+1}}-\bar{\omega}_{\tau_{i}}\right)\right]
$$

can be uniquely extended to $M^{\rho^{*}}$ by continuity. Using the same notation for its extension, we can replace the optimization variables in (3.10) by taking " $F \in M^{\rho^{*}}$ " without changing the value of the optimization problem.

Proof. By [RR91, Ch. III.3.4, Proposition 3], we have (B.1) under the given hypotheses; the measure considered being $d \nu \times d t$, the sigma-algebra being the $\mathcal{G}$-previsible one, and the Young function being $\rho^{*}$. One need only observe that the previsible sigma-algebra is generated by the algebra of sets whose elements are finite disjoint unions of "base" sets of the form $D \times(\underline{\tau}, \bar{\tau}]$, with $D \in \mathcal{G}_{\underline{\tau}}$ and $\mathcal{G}$-stopping times $\underline{\tau} \leq \bar{\tau}$. If we denote by $v$ the value of problem (3.10), from now on assumed finite, we then have for all $F \in S_{a}(\mathcal{G}), \beta>0$ :

$$
\mathbb{E}^{\nu}\left[\int_{0}^{T}\left(F_{t}(\bar{\omega}) / \beta\right) d \bar{\omega}_{t}\right] \leq v+\mathbb{E}^{\nu}\left[\int_{0}^{T} \rho^{*}\left(F_{t} / \beta\right) d t\right],
$$

so by definition

$$
\mathbb{E}^{\nu}\left[\int_{0}^{T} F_{t}(\bar{\omega}) d \bar{\omega}_{t}\right] \leq(v+1)\|F\|_{\rho^{*}} .
$$

This shows that the discrete integral, seen as a continuous linear functional on $S_{a}(\mathcal{G})$, can be uniquely and continuously extended to the norm closure of this space, which we know to coincide with $M^{\rho^{*}}$. Because the convex functional $F \mapsto \mathbb{E}^{\nu}\left[\int_{0}^{T} \rho^{*}\left(F_{t}\right) d t\right]$ is finite throughout $M^{\rho^{*}}$, for all $F, \bar{F} \in M^{\rho^{*}}$ with $\|F-\bar{F}\|_{\rho^{*}} \leq 1 / 2$ we have that

$$
\mathbb{E}^{\nu}\left[\int_{0}^{T} \rho^{*}\left(\bar{F}_{t}\right) d t\right] \leq 1 / 2 \mathbb{E}^{\nu}\left[\int_{0}^{T} \rho^{*}\left(2 F_{t}\right) d t\right]+1 / 2 \mathbb{E}^{\nu}\left[\int_{0}^{T} \rho^{*}\left(\frac{F_{t}-\bar{F}_{t}}{1 / 2}\right) d t\right] \leq c_{F}+1,
$$

where $c_{F}$ is a constant only depending on $F$. This shows that the convex functional is locally bounded and thus continuous by classical results; the last statement then follows.

Lemma B.3. Assume that $\rho^{*}$ (equiv. $\rho$ ) is an $N$-function, that $\rho$ is in $\Delta_{2}$, and that the refined dual (3.10) is finite. Then (3.10) is attained in $M^{\rho^{*}}$.

Proof. By Lemma B.2, we can consider (3.10) as defined over $M^{\rho^{*}}$. By [RR91, Ch. IV.4.1, Theorem 6] we see that $L^{\rho^{*}}$ is the norm dual space of $M^{\rho}$, so the classical Banach-Alaoglu's theorem implies that closed balls in $M^{\rho^{*}}$ are $\sigma\left(M^{\rho^{*}}, M^{\rho}\right)$-compact, since $M^{\rho^{*}}$ is a norm-closed 
and convex subset of $L^{\rho^{*}}$. By [RR91, Ch. V.5.3, Theorem 3] and the comment following its proof, we see that $\rho$ in $\Delta_{2}$ implies that

$$
\lim _{\|F\|_{\rho^{*} \rightarrow \infty}} \frac{\mathbb{E}^{\nu}\left[\int_{0}^{T} \rho^{*}\left(F_{t}\right) d t\right]}{\|F\|_{\rho^{*}}}=+\infty
$$

As a consequence of this and (B.2) (which holds also in $M^{\rho^{*}}$ ), we get

$$
\mathbb{E}^{\nu}\left[\int_{0}^{T} F_{t}(\bar{\omega}) d \bar{\omega}_{t}-\int_{0}^{T} \rho^{*}\left(F_{t}(\bar{\omega})\right) d t\right] \leq\|F\|_{\rho^{*}}\left\{v+1-\frac{\mathbb{E}^{\nu}\left[\int_{0}^{T} \rho^{*}\left(F_{t}\right) d t\right]}{\|F\|_{\rho^{*}}}\right\}
$$

where $v$ is the value of (3.10), and by (B.3) the r.h.s. above goes to $-\infty$ as $\|F\|_{\rho^{*}} \rightarrow \infty$. This shows that in computing (3.10) one may restrict the problem to a big enough fixed ball in $M^{\rho^{*}}$, which is $\sigma\left(M^{\rho^{*}}, M^{\rho}\right)$-compact. As at the end of the proof of Lemma B.2, we observe that the objective function is norm-continuous, and because it is concave it is also $\sigma\left(M^{\rho^{*}}, M^{\rho}\right)$-upper semicontinuous. The existence of an optimizer in $M^{\rho^{*}}$ follows.

Lemma B.4. Suppose (3.10) is finite and attained by some $\hat{F} \in M^{\rho^{*}}$, and that $\rho^{*}$ is a differentiable $N$-function (equiv. $\rho$ is a strictly convex $N$-function) which is in $\Delta_{2}$. Setting

$$
\alpha_{t}(\bar{\omega}):=\left(\rho^{*}\right)^{\prime}\left(\hat{F}_{t}(\bar{\omega})\right) \text { and } \xi_{t}(\bar{\omega}):=\bar{\omega}_{t}-\int_{0}^{t} \alpha_{t}(\bar{\omega}) d t
$$

we have that $\mathbb{E}^{\nu}\left[\int_{0}^{T} \rho\left(\alpha_{t}\right) d t\right]<+\infty$, and so $\alpha \in L^{\rho}$ and $\xi$ is a $(\nu, \mathcal{G})$-martingale.

If further $\nu \ll \gamma$, then $\xi$ is a $(\nu, \mathcal{G})$-Brownian motion, $(\xi, i d)_{\#} \nu \in \Pi^{\mathcal{F}, \mathcal{G}}(\gamma, \nu)$, the primal problem (3.6) is finite and its value is equal to (3.9) and (3.10).

Proof. We first observe that by the identity of sub-differentials

$$
\mathbb{E}^{\nu}\left[\int_{0}^{T} \rho \circ\left(\rho^{*}\right)^{\prime}\left(\hat{F}_{t}\right) d t\right]=-\mathbb{E}^{\nu}\left[\int_{0}^{T} \rho^{*}\left(\hat{F}_{t}\right) d t\right]+\mathbb{E}^{\nu}\left[\int_{0}^{T} \hat{F}_{t}\left(\rho^{*}\right)^{\prime}\left(\hat{F}_{t}\right) d t\right],
$$

so the finiteness of the l.h.s. is equivalent to the finiteness of the second term in the r.h.s. Since $\rho^{*}$ is an N-function in $\Delta_{2}$ we have by (A.1) that, when $\hat{F}_{t}(\bar{\omega})$ is large, the integrand $\hat{F}_{t}(\bar{\omega})\left(\rho^{*}\right)^{\prime}\left(\hat{F}_{t}(\bar{\omega})\right)$ is dominated by a (fixed) constant times $\rho^{*}\left(\hat{F}_{t}(\bar{\omega})\right)$, and so we conclude that the left- and righthand sides above are indeed finite. In particular $\alpha \in L^{\rho}$ holds, and $h \in M^{\rho^{*}} \mapsto \mathbb{E}^{\nu}\left[\int_{0}^{T} \alpha_{t} h_{t} d t\right]$ is finite-valued and continuous by [RR91, Ch. III.3.3, Proposition 1].

Since (3.10) is a concave problem, we have that if $\hat{\zeta}$ is an optimizer for $\sup _{\zeta \in M^{\rho^{*}}} H(\zeta)$, then $\forall h \in M^{\rho^{*}}$ it holds that $\left.\frac{\partial}{\partial \varepsilon} H(\hat{\zeta}+\varepsilon h)\right|_{\varepsilon=0}=0$, where

$$
H(\zeta):=\mathbb{E}^{\nu}\left[\int_{0}^{T} \zeta_{t}(\bar{\omega}) d \bar{\omega}_{t}\right]-\mathbb{E}^{\nu}\left[\iint_{0}^{T} \rho^{*}\left(\zeta_{t}\right) d t\right] .
$$

Thus we get that

$$
\mathbb{E}^{\nu}\left[\int_{0}^{T} h_{t}(\bar{\omega}) d \bar{\omega}_{t}\right]-\mathbb{E}^{\nu}\left[\int_{0}^{T} \alpha_{t} h_{t} d t\right]=0 \quad \forall h \in M^{\rho^{*}} .
$$

This means that $\mathbb{E}^{\nu}\left[\int_{0}^{T} h_{t} d \xi_{t}\right]=0$ for all such $h$, which implies that $\xi$ is indeed a $(\nu, \mathcal{G})$ martingale. Since the bracket of the canonical process is the identity under $\gamma$, this is inherited by $\xi$ by Girsanov theorem under the assumption $\nu \ll \gamma$, so by Levy's theorem $\xi$ is then a $(\nu, \mathcal{G})$-Brownian motion. By Lemma 2.6, $(\xi, i d)_{\#} \nu$ is causal. In light of the finiteness in (B.4), this proves that the primal problem (3.6) is finite. The lower semicontinuity of $(\omega, \bar{\omega}) \mapsto \int_{0}^{T} \rho\left((\widetilde{\overline{\omega-\omega}})_{t}\right) d t$ was established in Lemma A.1, and so by Theorem 5.6 we get that 
there is no duality gap. To conclude the proof, we only need to check the equality between (3.6) and the refined dual. For this, we rewrite (B.4) as

$$
\mathbb{E}^{\nu}\left[\int_{0}^{T} \rho\left(\alpha_{t}\right) d t\right]=-\mathbb{E}^{\nu}\left[\int_{0}^{T} \rho^{*}\left(\hat{\zeta}_{t}\right) d t\right]+\mathbb{E}^{\nu}\left[\int_{0}^{T} \hat{\zeta}_{t}(\bar{\omega}) d \bar{\omega}_{t}\right]
$$

where we used that $\mathbb{E}^{\nu}\left[\int_{0}^{T} \hat{\zeta}_{t} d \xi_{t}\right]=0$. This proves that the refined dual has a greater value than the primal, and we conclude by Lemma B.1.

We can finally give the proof of Theorem 3.8:

Proof of Theorem 3.8. Under the assumptions made, both $\rho$ and $\rho^{*}$ are $\mathrm{N}$-functions in $\Delta_{2}$. For Point $(i)$, and thanks to Lemma B.1, we only need to prove that when the refined dual is finite, its value coincides with the primal one. This follows by Lemmata B.3 and B.4. Point (ii) is contained in Lemma B.2. Point (iii) follows from the latter lemma, and because the strict convexity of $\rho$ implies differentiability of $\rho^{*}$. Finally, Point $(i v)$ is given by Lemma B.4 together with an application of Girsanov theorem.

We stress that Point (iv) of Theorem 3.8 can also be obtained via more sophisticated stochastic analysis arguments: if the primal problem (equiv. the refined dual) is finite, then as in the proof of Lemma B.2 one shows that for all $F \in S_{a}(\mathcal{G}): \mathbb{E}^{\nu}\left[\int_{0}^{T} F_{t}(\bar{\omega}) d \bar{\omega}_{t}\right] \leq(v+1)\|F\|_{\rho^{*}}$, where $v$ is the common optimal value. If $|F| \leq C$ then $\|F\|_{\rho^{*}} \leq C\|1\|_{\rho^{*}}<\infty$. This suggests that $\left\{\int_{0}^{T} F_{t}(\bar{\omega}) d \bar{\omega}_{t}: F \in S_{a}(\mathcal{G}),\|F\|_{\infty} \leq 1\right\}$ is bounded in $\nu$-probability, so by the BichtelerDellacherie theorem $\bar{\omega}$ is a $(\nu, \mathcal{G})$-semimartingale, and we conclude by Girsanov theorem. Such a proof is reminiscent of original arguments in [Jac85].

\section{Projections of processes}

We recall the notions of (dual) optional and predictable projections, which are used throughout the article, and refer to [DM80, Ch. VI] for an accurate study of the subject.

Let $X$ be a positive or bounded measurable process on a filtered probability space $(\Omega, \mathcal{H}, \mathbb{P})$. The optional projection of $X$ is the unique (up to indistinguishability) optional process $Y$ such that

$$
\mathbb{E}\left[X_{\tau} \mathbb{I}_{\{\tau<\infty\}} \mid \mathcal{H}_{\tau}\right]=Y_{\tau} \mathbb{I}_{\{\tau<\infty\}} \text { a.s. }
$$

for every stopping time $\tau$. The predictable projection of $X$ is the unique (up to indistinguishability) predictable process $N$ such that

$$
\mathbb{E}\left[X_{\tau} \mathbb{I}_{\{\tau<\infty\}} \mid \mathcal{H}_{\tau-}\right]=N_{\tau} \mathbb{I}_{\{\tau<\infty\}} \text { a.s. }
$$

for every predictable stopping time $\tau$. These notions of projection can be given for a broader class of processes, including those of integrable variation; see [DM80, Remark VI.44-(f)].

Now, let $H$ be a raw process of integrable variation on $(\Omega, \mathcal{H}, \mathbb{P})$. The dual optional (resp. predictable) projection of $H$ is the optional (resp. predictable) integrable variation process $U$ defined by

$$
\mathbb{E}\left[\int_{0}^{\infty} X_{t} d U_{t}\right]=\mathbb{E}\left[\int_{0}^{\infty} X_{t} d H_{t}\right]
$$

for any bounded optional (resp. predictable) process $X$. W.l.o.g. we assume $U_{0}=0$.

The following lemma is fundamental for the proof of Theorem 3.1 and Proposition 4.4. It follows directly from [DM80, Lemma 7, App. I], and holds for any two filtrations $\mathcal{F}$ and $\mathcal{G}$ on $\mathcal{C}$. 
Lemma C.1. Let $\pi \in \Pi(\mu, \nu)$ be a (non-necessarily causal) transport plan, and let $\Lambda$ be a $\left(\mathcal{B}([0, T]) \otimes \mathcal{F}_{T} \otimes \mathcal{G}_{T}\right)$-measurable process on $\mathcal{C} \times \mathcal{C}$ of integrable variation. Then:

1. The optional projection of $\Lambda$ with respect to $\left(\pi,{ }^{\pi}(\mathcal{F} \otimes\{\emptyset, \mathcal{C}\})\right)\left(\right.$ resp. $\left.\left(\pi,{ }^{\pi}(\{\emptyset, \mathcal{C}\} \otimes \mathcal{G})\right)\right)$, which we denote by ${ }^{o} \Lambda^{\mathcal{F}}$ (resp. ${ }^{o} \Lambda^{\mathcal{G}}$ ) is $\pi$-indistinguishable from an optional process with respect to $\left(\mu,{ }^{\mu} \mathcal{F}\right)$ (resp. $\left.\left(\nu,{ }^{\nu} \mathcal{G}\right)\right)$, so w.l.o.g. one may assume

$$
{ }^{o} \Lambda^{\mathcal{F}}(\omega, \bar{\omega})={ }^{o} \Lambda^{\mathcal{F}}(\omega), \quad{ }^{o} \Lambda^{\mathcal{G}}(\omega, \bar{\omega})={ }^{o} \Lambda^{\mathcal{G}}(\bar{\omega}) .
$$

The analogous statement holds for the predictable projections.

2. The dual optional projection of $\Lambda$ with respect to $\left(\pi,{ }^{\pi}(\mathcal{F} \otimes\{\emptyset, \mathcal{C}\})\right)\left(\right.$ resp. $\left.\left(\pi,{ }^{\pi}(\{\emptyset, \mathcal{C}\} \otimes \mathcal{G})\right)\right)$, which we denote by ${ }^{\mathcal{F}} \Lambda^{o}$ (resp. $\left.{ }^{\mathcal{G}} \Lambda^{o}\right)$, is $\pi$-indistinguishable from an optional process with respect to $\left(\mu,{ }^{\mu} \mathcal{F}\right)$ (resp. $\left.\left(\nu,{ }^{\nu} \mathcal{G}\right)\right)$, so w.l.o.g. one may assume

$$
{ }^{\mathcal{F}} \Lambda^{o}(\omega, \bar{\omega})={ }^{\mathcal{F}} \Lambda^{o}(\omega), \quad{ }^{\mathcal{G}} \Lambda^{o}(\omega, \bar{\omega})={ }^{\mathcal{G}} \Lambda^{o}(\bar{\omega}) .
$$

The analogous statement holds for the dual predictable projections.

The essential difference between ${ }^{o} \Lambda^{\mathcal{F}}$ and ${ }^{\mathcal{F}} \Lambda^{o}$, as explained in [DM80, Remark VI.74-(c)], is that while the first one formalizes $\mathbb{E}\left[\Lambda_{t} \mid \mathcal{F}_{t}\right]$, the second one does so to $\int_{0}^{t} \mathbb{E}\left[d \Lambda_{s} \mid \mathcal{F}_{s}\right]$. Causality imposes a strong relation between the two kinds of projections:

$$
\begin{aligned}
{ }^{\mathcal{F}} \Lambda_{t}^{o}-{ }^{\mathcal{F}} \Lambda_{0}^{o} & =\int_{0}^{t} \mathbb{E}^{\pi}\left[d \Lambda_{s} \mid \mathcal{F}_{s}\right]=\int_{0}^{t} \mathbb{E}^{\pi}\left[d \Lambda_{s} \mid \mathcal{F}_{t}\right]=\mathbb{E}^{\pi}\left[\int_{0}^{t} d \Lambda_{s} \mid \mathcal{F}_{t}\right] \\
& =\mathbb{E}^{\pi}\left[\Lambda_{t}-\Lambda_{0} \mid \mathcal{F}_{t}\right]={ }^{o} \Lambda_{t}^{\mathcal{F}}-{ }^{o} \Lambda_{0}^{\mathcal{F}}
\end{aligned}
$$

This result is formalised in Lemma C.2, and was crucial in our applications to optimal stopping problems in Section 4.1. Such a phenomenon is not symmetric, just as causality, i.e., one does not expect it to hold for projections w.r.t. $\mathcal{G}$. We give a proof of such result for completeness. However, one can see it also as a consequence of results in [AL16]. Indeed, in that article it is proved that if two filtrations $\mathcal{H}^{1} \subseteq \mathcal{H}^{2}$ satisfy the $H$-hypothesis, then for any $\mathcal{H}^{2}$-optional process of integrable variation, its $\mathcal{H}^{1}$-optional and $\mathcal{H}^{1}$-dual optional projections coincide. Thanks to Remark 2.3, Lemma C.2 then follows by [AL16, Theorem 2].

Lemma C.2. Let $\pi \in \Pi^{\mathcal{F}, \mathcal{G}}(\mu, \nu)$ be a causal transport plan, and let $\Lambda$ be an $(\mathcal{F} \otimes \mathcal{G})$-adapted, integrable variation càdlàg process with $\Lambda_{0}=0$. Then

$$
{ }^{\mathcal{F}} \Lambda^{o} \text { is } \pi \text {-indistinguishable from }{ }^{o} \Lambda^{\mathcal{F}} .
$$

Proof. We drop the superscript $\mathcal{F}$ to simplify the notation. Fix $t \in[0, T]$, and consider the process $X=\left(X_{s}\right)_{s \in[0, t]}$ with constant paths given by

$$
X_{s}=\mathbb{I}_{\left\{{ }^{o} \Lambda_{t}>\Lambda_{t}^{o}\right\}}, \quad s \in[0, t] .
$$

Its optional projection with respect to $\left(\pi,{ }^{\pi}(\mathcal{F} \otimes\{\emptyset, \mathcal{C}\})\right)$ satisfies

$$
{ }^{o} X_{s}=\pi\left[{ }^{o} \Lambda_{t}>\left.\Lambda_{t}^{o}\right|^{\pi}\left(\mathcal{F}_{s} \otimes\{\emptyset, \mathcal{C}\}\right)\right], \quad s \in[0, t] .
$$

Note that $\left({ }^{o} X_{s}\right)_{s \in[0, t]}$ is an ${ }^{\pi}(\mathcal{F} \otimes\{\emptyset, \mathcal{C}\})$-martingale, hence $\pi$-indistinguishable from an $(\mathcal{F} \otimes$ $\{\emptyset, \mathcal{C}\})$-martingale, by [DM80, Lemma 7, App. I], which is then also an $(\mathcal{F} \otimes \mathcal{G})$-martingale by causality; see Remark 2.3. This means that $\left({ }^{o} X_{s}\right)_{s \in[0, t]}$ is the càdlàg version of the $(\mathcal{F} \otimes \mathcal{G})$ martingale $M_{s}=\pi\left[{ }^{o} \Lambda_{t}>\Lambda_{t}^{o} \mid \mathcal{F}_{s} \otimes \mathcal{G}_{s}\right], 0 \leq s \leq t$, thus $\pi$-indistinguishable from the optional projection of $X$ w.r.t. $\left(\pi,{ }^{\pi}\{\mathcal{F} \otimes \mathcal{G}\}\right)$, which we denote by $Y$. 
Now, by definition of optional projection, $\mathbb{E}^{\pi}\left[\Lambda_{t} X_{t}\right]=\mathbb{E}^{\pi}\left[{ }^{0} \Lambda_{t} X_{t}\right]$. On the other hand, since $X$ is constant and $\Lambda_{0}=0$,

$$
\mathbb{E}^{\pi}\left[\Lambda_{t} X_{t}\right]=\mathbb{E}^{\pi}\left[\Lambda_{t} X_{0}\right]=\mathbb{E}^{\pi}\left[\int_{0}^{t} X_{s} d \Lambda_{s}\right]
$$

Now, using [DM80, Remark VI.58-(d)] and the fact that $\Lambda$ is an $(\mathcal{F} \otimes \mathcal{G})$-adapted integrable variation process, we have that $\mathbb{E}^{\pi}\left[\int_{0}^{t} X_{s} d \Lambda_{s}\right]=\mathbb{E}^{\pi}\left[\int_{0}^{t} Y_{s} d \Lambda_{s}\right]$. Therefore we have

$$
\begin{aligned}
\mathbb{E}^{\pi}\left[\Lambda_{t} X_{t}\right] & =\mathbb{E}^{\pi}\left[\int_{0}^{t} Y_{s} d \Lambda_{s}\right]=\mathbb{E}^{\pi}\left[\int_{0}^{t} o X_{s} d \Lambda_{s}\right]=\mathbb{E}^{\pi}\left[\int_{0}^{t} X_{s} d \Lambda_{s}^{o}\right] \\
& =\mathbb{E}^{\pi}\left[\Lambda_{t}^{o} X_{t}\right]-\mathbb{E}^{\pi}\left[\Lambda_{0}^{o} X_{t}\right]=\mathbb{E}^{\pi}\left[\Lambda_{t}^{o} X_{t}\right]
\end{aligned}
$$

where in the second equality we use that ${ }^{\circ} X$ and $Y$ are $\pi$-indistinguishable, and in the third one the definition of dual optional projection (recall that $\Lambda_{0}^{o}=0$ ). Altogether we have

$$
\mathbb{E}^{\pi}\left[{ }^{o} \Lambda_{t} \mathbb{I}_{\left\{{ }^{o} \Lambda_{t}>\Lambda_{t}^{o}\right\}}\right]=\mathbb{E}^{\pi}\left[\Lambda_{t}^{o o} \mathbb{I}_{\left\{{ }^{o} \Lambda_{t}>\Lambda_{t}^{o}\right\}}\right],
$$

hence $\left\{{ }^{o} \Lambda_{t}>\Lambda_{t}^{o}\right\}$ is $\pi$-negligible. Arguing similarly, we get that $\left\{{ }^{o} \Lambda_{t} \neq \Lambda_{t}^{o}\right\}$ is $\pi$-negligible. Since this is true for all $t \in[0, T]$, we have that ${ }^{\circ} \Lambda$ and $\Lambda^{o}$ are versions of each other and hence $\pi$-indistinguishable since both are càdlàg (see [DM80, Theorem VI.47]).

\section{References}

[ABC18] B. Acciaio, J. Backhoff-Veraguas, and R. Carmona, Extended mean field control problems: stochastic maximum principle and transport perspective, arXiv:1802.05754 (2018).

[ADI06] S. Ankirchner, S. Dereich, and P. Imkeller, The Shannon information of filtrations and the additional logarithmic utility of insiders, Ann. Probab. 34 (2006), no. 2, 743-778. MR 2223957

[ADI07] , Enlargement of filtrations and continuous Girsanov-type embeddings, Séminaire de Probabilités XL, Lecture Notes in Math., vol. 1899, Springer, Berlin, 2007, pp. 389-410. MR 2409018

[AIS98] J. Amendinger, P. Imkeller, and M. Schweizer, Additional logarithmic utility of an insider, Stochastic Processes and their Applications 75 (1998), no. 2, 263-286.

[AJ17] A. Aksamit and M. Jeanblanc, Enlargement of filtration with finance in view, Springer, 2017.

[AL16] A. Aksamit and L. Li, Projections, pseudo-stopping times and the immersion property, Séminaire de Probabilités XLVIII, Springer, 2016, pp. 459-467.

[Ald81] D. Aldous, Weak convergence and the general theory of processes (weak convergence of stochastic processes for processes viewed in the Strasbourg manner), unpublished, 1981.

[BBLZ17] J. Backhoff-Veraguas, M. Beiglbock, Y. Lin, and A. Zalashko, Causal transport in discrete time and applications, SIAM Journal on Optimization 27 (2017), no. 4, $2528-2562$. 
[BC77] J. R. Baxter and R. V. Chacon, Compactness of stopping times, Z. Wahrscheinlichkeitstheorie und Verw. Gebiete 40 (1977), no. 3, 169-181. MR 0517871

$\left[\mathrm{BCC}^{+} 15\right]$ J.-D. Benamou, G. Carlier, M. Cuturi, L. Nenna, and G. Peyré, Iterative Bregman projections for regularized transportation problems, SIAM Journal on Scientific Computing 37 (2015), no. 2, A1111-A1138.

[BCH17] M. Beiglböck, A.M.G. Cox, and M. Huesmann, Optimal transport and Skorokhod embedding, Inventiones mathematicae 208 (2017), no. 2, 327-400.

[BG19] M. Beiglböck and C. Griessler, A land of monotone plenty, no. 1, 109-127, Annali della SNS.

[BHLP13] M. Beiglböck, P. Henry-Labordére, and F. Penkner, Model-independent bounds for option prices - a mass transport approach, Finance Stoch. 17 (2013), no. 3, 477-501.

[BNT19] J. Bion-Nadal and D. Talay, On a Wasserstein-type distance between solutions to stochastic differential equations, The Annals of Applied Probability 29 (2019), no. 3, 1609-1639.

[BS11] M. Beiglböck and W. Schachermayer, Duality for Borel measurable cost functions, Trans. Amer. Math. Soc. 363 (2011), no. 8, 4203-4224. MR 2792985 (2012k:49108)

[BY78] P. Brémaud and M. Yor, Changes of filtrations and of probability measures, Zeitschrift für Wahrscheinlichkeitstheorie und verwandte Gebiete 45 (1978), no. 4, 269-295.

[CJ18] D. Coculescu and M. Jeanblanc, Structure conditions under short-sales constraints and applications to converging asset prices, 2018.

[CK92] J. Cvitanić and I. Karatzas, Convex duality in constrained portfolio optimization, Ann. Appl. Probab. 2 (1992), no. 4, 767-818. MR 1189418

[CT07] F. Coquet and S. Toldo, Convergence of values in optimal stopping and convergence of optimal stopping times, Electron. J. Probab. 12 (2007), no. 8, 207-228. MR 2299917

[Cut13] M. Cuturi, Sinkhorn distances: Lightspeed computation of optimal transport, Advances in neural information processing systems, 2013, pp. 2292-2300.

[DM80] C. Dellacherie and P.-A. Meyer, Probabilités et potentiel. Chapitres V à VIII, revised ed., Actualités Scientifiques et Industrielles, vol. 1385, Hermann, Paris, 1980, Théorie des martingales. [Martingale theory]. MR 566768

[EK18] S. Eckstein and M. Kupper, Computation of optimal transport and related hedging problems via penalization and neural networks, Applied Mathematics \& Optimization (2018), 1-29.

[FF96] J.P. Florens and D. Fougere, Noncausality in continuous time, Econometrica: Journal of the Econometric Society (1996), 1195-1212.

[FÜ04] D. Feyel and A. S. Üstünel, Monge-Kantorovitch measure transportation and MongeAmpère equation on Wiener space, Probab. Theory Related Fields 128 (2004), no. 3, 347-385. MR 2036490 (2004m:60121) 
[GHLT14] A. Galichon, P. Henry-Labordére, and N. Touzi, A stochastic control approach to no-arbitrage bounds given marginals, with an application to lookback options, The Annals of Applied Probability 24 (2014), no. 1, 312-336.

[Jac80] J. Jacod, Weak and strong solutions of stochastic differential equations, Stochastics 3 (1980), 171-191.

[Jac85] _ Grossissement initial, hypothèse ( $\left.H^{\prime}\right)$, et théorème de Girsanov, Grossissements de Filtrations: Exemples et Applications (T. Jeulin and M. Yor, eds.), Lecture Notes in Mathematics, vol. 1118, Springer, Berlin - Heidelberg, 1985, pp. 15-35.

[Jeu80] T. Jeulin, Semi-martingales et grossissement d'une filtration, Lecture Notes in Mathematics, vol. 833, Springer, Berlin, 1980. MR MR604176 (82h:60106)

[JY78] T. Jeulin and M. Yor, Grossissement d'une filtration et semi-martingales: formules explicites, Séminaire de Probabilités, XII (Univ. Strasbourg, Strasbourg, 1976/1977), Lecture Notes in Math., vol. 649, Springer, Berlin, 1978, pp. 78-97. MR MR519998

[JY79] _ Inégalité de Hardy, semimartingales, et faux-amis, Séminaire de probabilités de Strasbourg 13 (1979), 332-359 (fre).

[JYC09] M. Jeanblanc, M. Yor, and M. Chesney, Mathematical methods for financial markets, Springer Finance, Springer-Verlag London Ltd., London, 2009. MR 2568861

[Kan42] L. V. Kantorovich, On the transfer of masses, Dokl. Akad. Nauk. SSSR, vol. 37, 1942, pp. 227-229.

[Kel84] H. G. Kellerer, Duality theorems for marginal problems, Z. Wahrsch. Verw. Gebiete 67 (1984), no. 4, 399-432. MR 761565

[KLS87] I. Karatzas, J. P. Lehoczky, and S. E. Shreve, Optimal portfolio and consumption decisions for a "small investor" on a finite horizon, SIAM J. Control Optim. 25 (1987), no. 6, 1557-1586. MR 912456

[KLSX91] I. Karatzas, J. P. Lehoczky, S. E. Shreve, and G.-L. Xu, Martingale and duality methods for utility maximization in an incomplete market, SIAM J. Control Optim. 29 (1991), no. 3, 702-730. MR 1089152

[KP15] Y. Kchia and P. Protter, Progressive filtration expansions via a process, with applications to insider trading, Int. J. Theor. Appl. Finance 18 (2015), no. 4, 1550027, 48. MR 3358108

[Kur14] T. G. Kurtz, Weak and strong solutions of general stochastic models, Electron. Commun. Probab. 19 (2014), no. 58, 16. MR 3254737

[Las15] R. Lassalle, Causal transference plans and their Monge-Kantorovich problems, Submitted, arXiv:1303.6925.v2, 2015.

[Léo12] C. Léonard, Girsanov theory under a finite entropy condition, Séminaire de Probabilités XLIV, Lecture Notes in Math., vol. 2046, Springer, Heidelberg, 2012, pp. 429465. MR 2953359

[LP90] D. Lamberton and G. Pagès, Sur l'approximation des réduites, Ann. Inst. H. Poincaré Probab. Statist. 26 (1990), no. 2, 331-355. MR 1063754 
[Mon81] G. Monge, Mémoire sur la théorie des déblais et des remblais, Histoire de l'Académie royale des sciences de Paris (1781).

[MY06] R. Mansuy and M. Yor, Random times and enlargements of filtrations in a Brownian setting, Springer, 2006.

[PC19] G. Peyré and M. Cuturi, Computational optimal transport, Foundations and Trends in Machine Learning 11 (2019), no. 5-6, 355-607.

[Pfl09] G. Ch. Pflug, Version-independence and nested distributions in multistage stochastic optimization, SIAM Journal on Optimization 20 (2009), no. 3, 1406-1420.

[PK96] I. Pikovsky and I. Karatzas, Anticipative portfolio optimization, Adv. in Appl. Probab. 28 (1996), no. 4, 1095-1122. MR 1418248

[PP12] G. Ch. Pflug and A. Pichler, A distance for multistage stochastic optimization models, SIAM J. Optim. 22 (2012), no. 1, 1-23. MR 2902682

[PP14]__ Multistage stochastic optimization, Springer Series in Operations Research and Financial Engineering, Springer, Cham, 2014. MR 3288310

[Pro04] P. Protter, Stochastic integration and differential equations, 2.1 ed., Applications of Mathematics (New York), Springer-Verlag, Berlin, 2004. MR MR1037262 (91i:60148)

[RR91] M. Rao and Z. Ren, Theory of Orlicz spaces, Monographs and Textbooks in Pure and Applied Mathematics, vol. 146, Marcel Dekker, Inc., New York, 1991. MR 1113700

[Sio58] M. Sion, On general minimax theorems, Pacific J. Math. 8 (1958), 171-176. MR 0097026

[Vil03] C. Villani, Topics in optimal transportation, no. 58, American Mathematical Soc., 2003 .

[XS92] G.-L. Xu and S. E. Shreve, A duality method for optimal consumption and investment under short-selling prohibition. I. General market coefficients, Ann. Appl. Probab. 2 (1992), no. 1, 87-112. MR 1143394

[Yor85] M. Yor, Entropie d'une partition, et grossissement initial d'une filtration, Grossissements de filtrations: exemples et applications, Springer, 1985, pp. 45-58.

[Yor97] $\quad$ Some aspects of Brownian motion. Part II, Lectures in Mathematics ETH Zürich, Birkhäuser Verlag, Basel, 1997, Some recent martingale problems. MR 1442263

[YW71] T. Yamada and S. Watanabe, On the uniqueness of solutions of stochastic differential equations, Journal of Mathematics of Kyoto University 11 (1971), no. 1, 155-167.

[Zae15] D. Zaev, On the Monge-Kantorovich Problem with Additional Linear Constraints, Mat. Zametki 98 (2015), no. 5, 664-683. MR 3438523 